\title{
Innate Immune Signaling Contributes to Tubular Cell Senescence in the Glis2 Knockout Mouse Model of Nephronophthisis
}

Heng Jin, ${ }^{\dagger}$ Yan Zhang, ${ }^{\star \dagger}$ Dingxiao Liu, ${ }^{*}$ Shan Shanshan Wang, ${ }^{\S}$ Qiong Ding, ${ }^{*}$ Prerna Rastogi, ${ }^{\varpi}$ Madison Purvis, * Angela Wang, ${ }^{*}$ Sarah Elhadi, ${ }^{\|}$Chongyu Ren, ${ }^{* *}$ Chao Cao, ${ }^{* \dagger}$ Yanfen Chai, ${ }^{\dagger}$ Peter Igarashi, ${ }^{\dagger \dagger}$ Anton M. Jetten, ${ }^{* \star}$ Dongmei Lu, ${ }^{* *}$ and Massimo Attanasio*

From the Department of Internal Medicine, * University of Iowa Carver College of Medicine, Iowa City, Iowa; the Department of Emergency Medicine, ${ }^{\dagger}$ Tianjin Medical University General Hospital, Tianjin, China; the Department of Vascular Surgery, ${ }^{\ddagger}$ Second Xiangya Hospital, Central South University, Changsha, China; the Department of Hepatic Oncology, ${ }^{\S}$ Liver Cancer Institute, Zhongshan Hospital, Fudan University, Shanghai, China; the Department of Pathology, "University of Iowa, Iowa City, Iowa; the Division of Neprhology," Children's Hospital of Illinois, Peoria, Illinois; the Department of Internal Medicine, ** University of Texas Southwestern Medical Center, Dallas, Texas; the Department of Internal Medicine, ${ }^{\dagger \dagger}$ University of Minnesota, Minneapolis, Minnesota; and the Cell Biology Section, ${ }^{\ddagger}$ Division of Intramural Research, National Institute of Environmental Health Sciences, NIH, Research Triangle Park, North Carolina

Accepted for publication September 4, 2019.

Address correspondence to Massimo Attanasio, M.D., University of Iowa, 285 Newton Rd., CBRB, 3270B, Iowa City, IA 52242. E-mail: massimo-attanasio@uiowa.edu.

\begin{abstract}
Nephronophthisis (NPHP), the leading genetic cause of end-stage renal failure in children and young adults, is a group of autosomal recessive diseases characterized by kidney-cyst degeneration and fibrosis for which no therapy is currently available. To date, mutations in $>25$ genes have been identified as causes of this disease that, in several cases, result in chronic DNA damage in kidney tubular cells. Among such mutations, those in the transcription factor-encoding GLIS2 cause NPHP type 7. Loss of function of mouse Glis2 causes senescence of kidney tubular cells. Senescent cells secrete proinflammatory molecules that induce progressive organ damage through several pathways, among which NF- $\kappa$ B signaling is prevalent. Herein, we show that the NF- $\kappa$ B signaling is active in Glis2 knockout kidney epithelial cells and that genetic inactivation of the toll-like receptor (TLR)/IL-1 receptor or pharmacologic elimination of senescent cells (senolytic therapy) reduces tubule damage, fibrosis, and apoptosis in the Glis2 mouse model of NPHP. Notably, in Glis2, Tlr2 double knockouts, senescence was also reduced and proliferation was increased, suggesting that loss of TLR2 activity improves the regenerative potential of tubular cells in Glis2 knockout kidneys. Our results further suggest that a combination of TLR/IL-1 receptor inhibition and senolytic therapy may delay the progression of kidney disease in NPHP type 7 and other forms of this disease. (Am J Pathol 2020, 190: 176-189; https://doi.org/10.1016/j.ajpath.2019.09.013)
\end{abstract}

Nephronophthisis (NPHP), the most frequent monogenic cause of chronic renal failure during the first three decades of life, is a group of autosomal recessive diseases that are characterized by progressive cystic and fibrotic degeneration of the kidneys. ${ }^{1}$ From the genetic standpoint, NPHP is a heterogeneous disease, caused by mutations identified in $>25$ genes. ${ }^{2}$ Despite the genetic heterogeneity of NPHP, almost all forms share two common progressive features: interstitial fibrosis and kidney atrophy.

Chronic DNA damage in kidney tubular cells is common to at least six forms of NPHP, each caused by mutations in a distinct gene (ZNF423, CEP164, MRE11, NEK8, SDCCAG8, and CEP290). ${ }^{3-6}$ Loss of function of Glis2 causes DNA damage and activation of the replication stress

Supported by NIH grants 1R01DK090326 (M.A.) and Z01-ES-101585 (A.M.J.); the National Natural Science Foundation of China grant 81871593 (Y.C.); the National Institute of Environmental Health Sciences Intramural Research Program; and the Fundamental Research Funds for the Central Universities, China, grant 3332019127 (H.J.).

H.J., Y.Z., and D.L. contributed equally to this work.

Disclosures: None declared. 
A

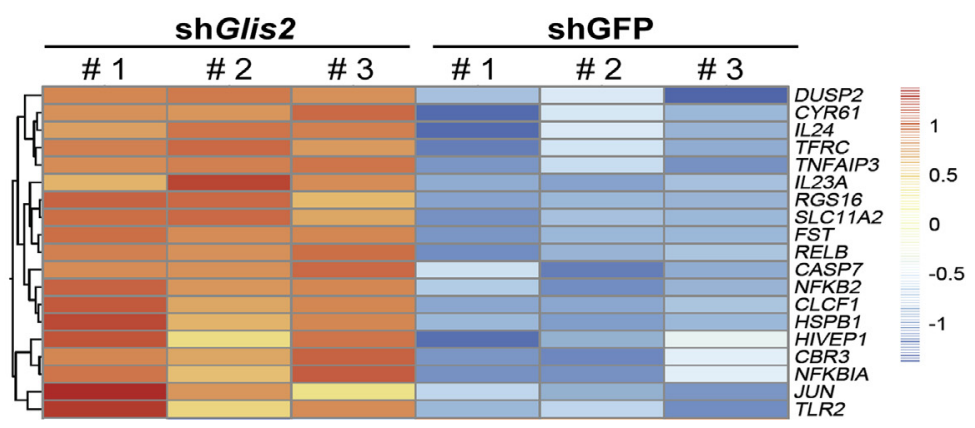

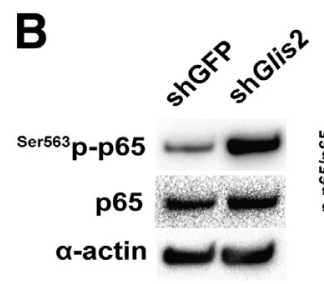

D

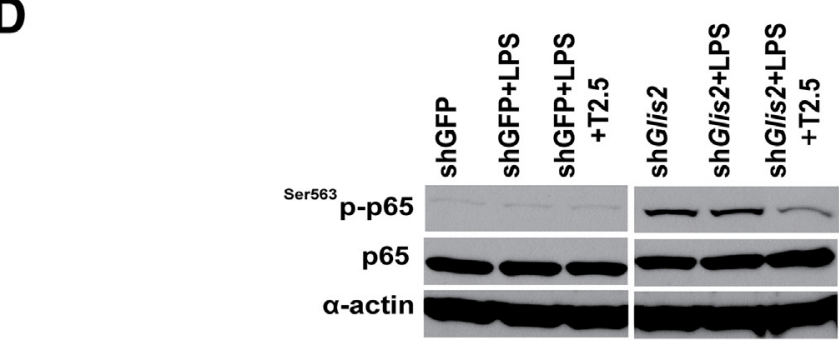

E
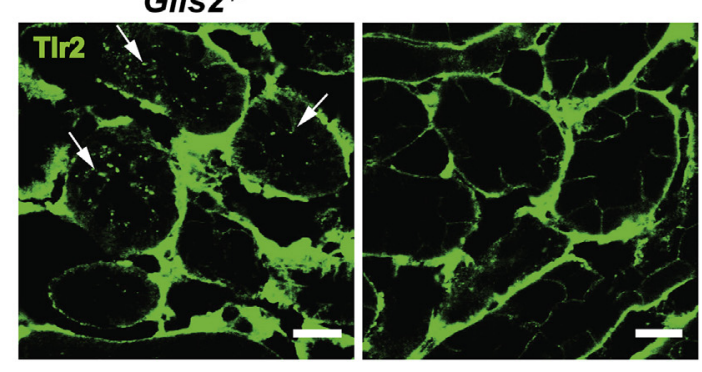

$\mathbf{F}$

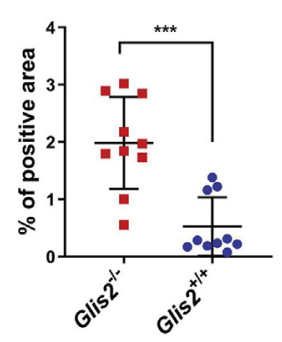

Figure 1 Toll-like receptor 2 (TLR2) signaling is activated in Glis2-knockdown kidney epithelial cells and in Glis2 knockout kidneys. A: Heat map showing RNA microarray expression profiles of NF$\kappa B$-related genes in immortalized kidney tubular cells in which Glis2 is silenced stably with Glis2targeted shRNA (shGlis2) or control cells in which the shRNA (shGFP) targets Aequorea victoria green fluorescence protein (GFP). Scale bar represents log of fold changes. B and C: Western blot analysis testing NF- $\mathrm{KB}$ activation in Glis2-knockdown immortalized kidney tubular cells versus controls (left panels) and correspondent quantification analysis by densitometry (right panels). Antibodies target total NF- $\kappa B$ p 65 and $\mathrm{I} \kappa \mathrm{B} \alpha$ and

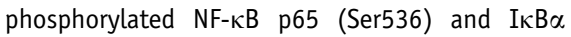
(Ser32). D: Western blot analysis testing effect of TLR2 blocking antibody (T2.5) on lipopolysaccharide (LPS) -mediated activation of NF- $\kappa B$ p65 in Glis2-knockdown tubular cells versus controls. E: Immunofluorescence confocal microscopy representative images of TLR2 expression in kidney sections from Glis2 knockout (Glis2 ${ }^{-1-}$ ) and wildtype $\left(\right.$ Glis $2^{+/+}$control) mice. Arrows indicate internalized (activated) TLR2. F: Correspondent quantification by digital image analysis of intracellular TLR2 signal. All $P$ values were calculated by two-tailed $t$-test. Data are expressed as means \pm SD (B and $\mathbf{C}$ ). $n=3$ samples per group $(\mathbf{A}-\mathbf{C}) ; n=10$ images per group $(\mathbf{F}) .{ }^{*} P<0.01$, $* * * P<0.001$. Scale bars $=20 \mu \mathrm{m}(\mathbf{E})$. response, resulting in extensive senescence of kidney epithelial cells in the mouse model of Nphp7.

This finding was of particular notice, providing a potential explanation for the link between DNA damage and progressive fibrosis in NPHP. Specifically, senescent cells are arrested in the cell cycle and secrete a wide spectrum of proinflammatory signaling molecules under the control of the proinflammatory and prosurvival pathway $\mathrm{NF}-\kappa \mathrm{B},{ }^{8-10}$ causing what is known as the senescence-associated secretory phenotype (SASP). Notably, this activity can lead to the induction of senescence in nonsenescent bystander cells, resulting in a loop that amplifies inflammation and organ damage. ${ }^{11,12}$ The inflammatory cytokine IL- $1 \alpha$ is of particular importance in mediating this phenomenon. It signals through its cognate receptor IL- 1 receptor (IL-1R), ${ }^{13}$ which is a member of the toll-like receptor (TLR)/IL-1R family. Like other members of this receptor family, such as TLR2 and TLR4, which are ubiquitously expressed, including in kidney epithelial cells, on stimulation IL-1R activates the NF- $\kappa \mathrm{B}$ pathway via common cellular adapter proteins, among which is myeloid differentiation primary response $88(\mathrm{Myd} 88){ }^{14,15}$

Multiple components of the SASP interact with receptors of the innate immune system, including IL-1R (IL-1 $\alpha / \mathrm{IL}-$ $1 \beta$ ), TLR4, and TLR2 (high-mobility group protein 1; other alarmins). Among the consequences of NF- $\kappa \mathrm{B}$ activation is the transcription of the TLR2 gene itself, resulting in amplification of the NF- $\mathrm{B}$ signaling. ${ }^{16,17}$ Given that DNA 
A

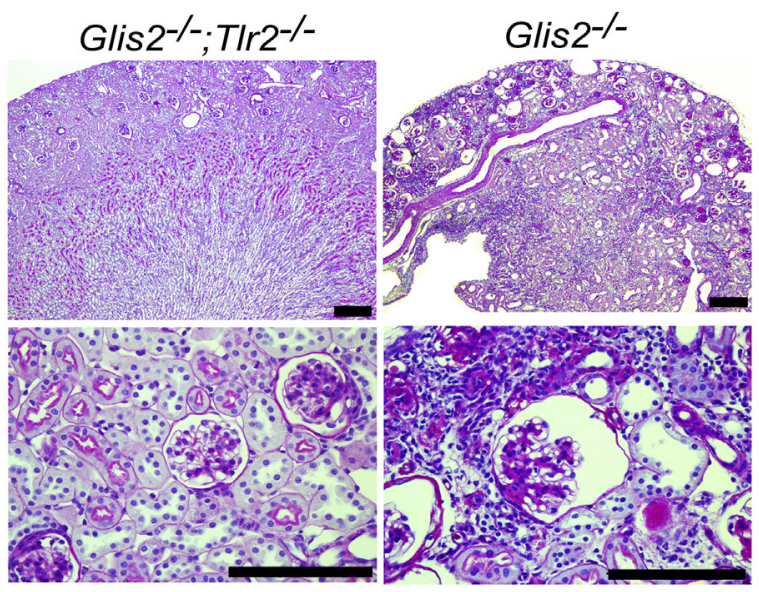

E

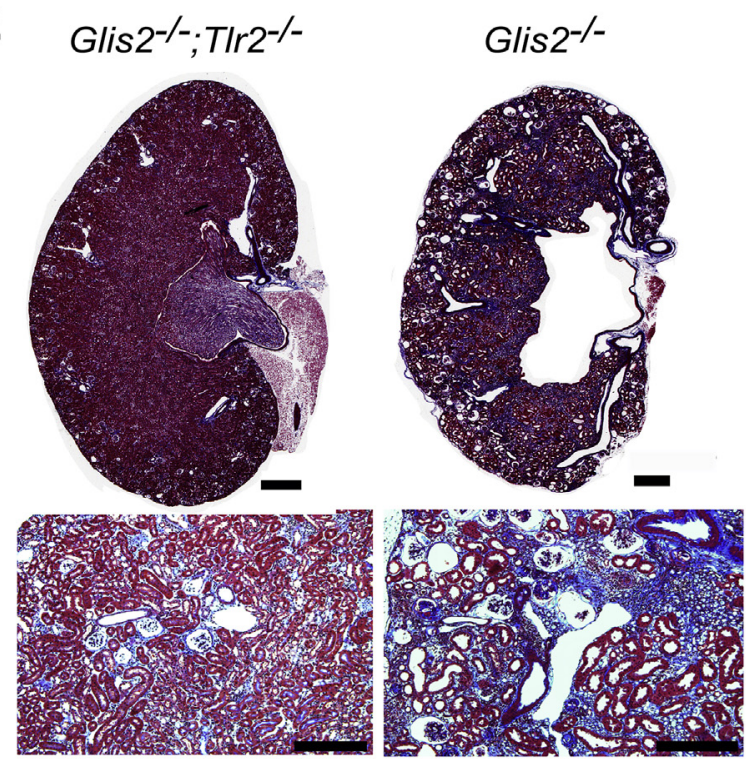

H Glis2 $2^{--} ;$TIr2 $^{-/}$

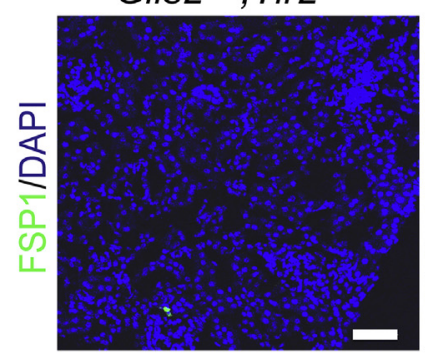

Glis2-/-

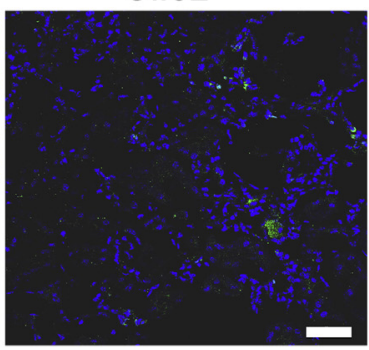

B

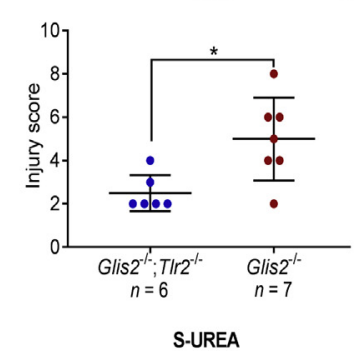

D

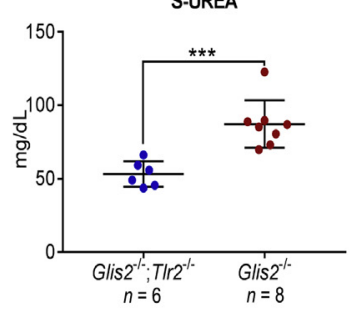

$\mathbf{F}$

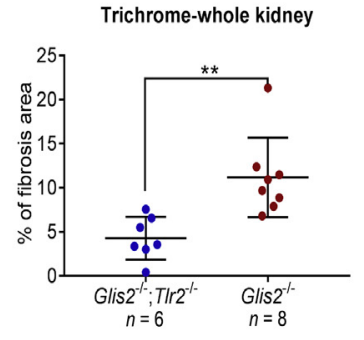

G

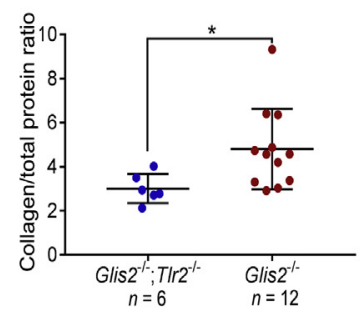

I Glis2 $\%:-T_{1 / 2} \%$
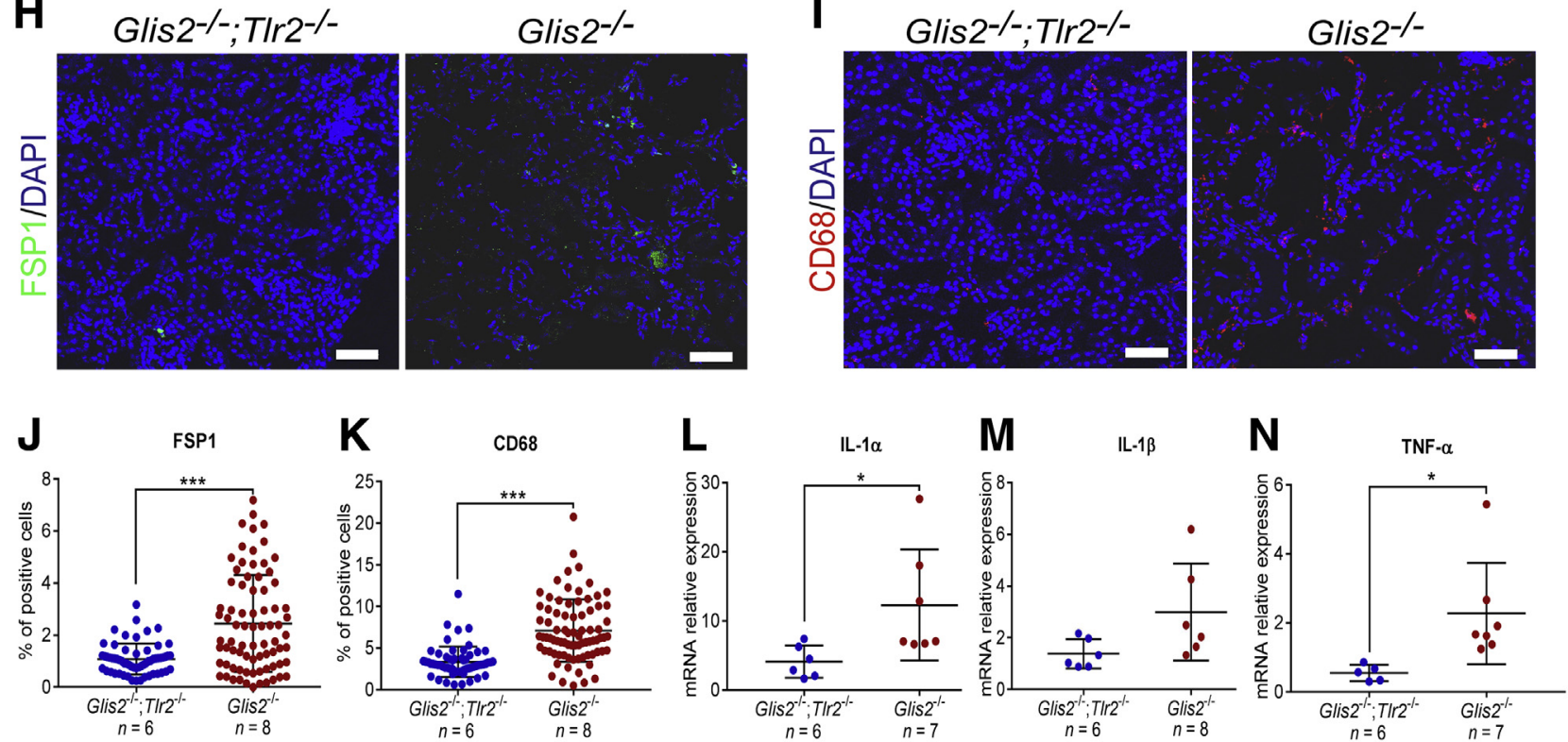
damage is known to activate NF- $\mathrm{BB},{ }^{18,19}$ we hypothesized that unprogrammed nuclear events triggered by loss-offunction mutations in Glis2 might be the primary causes of NF- $\kappa \mathrm{B}$ activation in kidney epithelial cells and that such activation might be amplified by subsequent increases in signaling via IL-1R, TLR2, and TLR4.

We examined the contribution of TLRs/IL-1R signaling to kidney damage, inflammation, and senescence in Glis2 knockout mice. Two mouse strains were generated: a double-knockout strain lacking both Glis2 and Tlr2 in all tissues $\left(\right.$ Glis $\left.2^{-1-} ; T l r 2^{-1-}\right)$ and a strain in which a kidneyspecific promoter $(\mathrm{Ksp})^{20}$ is used to inactivate Myd88 in tubular cells of Glis2-null mice (Glis $2^{-/-}$; ${ }^{\mathrm{KspCre}} M y d 88^{\mathrm{f} / \mathrm{f}}$ ). Both constitutive inactivation of Tlr2 and tubular cell-specific conditional deletion of Myd88 were associated with reduced kidney damage, fibrosis, DNA damage, and apoptosis in Glis2 knockout mice. Moreover, in the double-knockout mice, tubular cell senescence was decreased and proliferation was increased. Pharmacologic elimination of senescent cells in Glis2 knockout mice resulted in a similar outcome in terms of kidney damage, fibrosis, and apoptosis, but cell senescence and proliferation were not affected.

\section{Materials and Methods}

\section{Microarray-Based Expression and Gene Ontology Analysis}

The immortalized kidney epithelial cell lines that were stably transduced with inducible Glis2-targeting shRNA or the shRNA targeting green fluorescent protein (control) are described elsewhere. ${ }^{7}$ The efficiency of the Glis2 knockdown was confirmed by quantitative real-time PCR (>80\%). RNA was extracted from the induced Glis2-targeting shRNA line and shRNA targeting green fluorescent protein using Trizol (Sigma, St. Louis, MO), incubated with DNAse for 1 hour, and used to probe the Illumina Mouse WG-6_V2_0_R0_11278593_A beadchip (Illumina, San Diego, CA), according to the manufacturer's instructions. Ingenuity software version 8.6 (Qiagen Bioinformatics, Hilden, Germany) was used for gene ontology analysis. Raw data are retrievable on the Gene Expression Omnibus website (https://www.ncbi.nlm.nih.gov/geo; accession number GSE134338).

\section{Animal Studies}

All mice were on a C57/B6 background. The Glis $2^{-1-}$ mice were previously described. ${ }^{21}$ The KspCre mice ${ }^{20}$ were obtained from the O'Brien Kidney Center at the UT Southwestern Medical Center (Dallas, TX). Tlr2 knockout mice and $M y d 88^{f / f l}$ conditional mice were purchased from the Jax Laboratories (Bar Harbor, ME; stock numbers 4650 and 8888, respectively). All procedures were approved by the Institutional Animal Care and Use Committee of the University of Iowa (Iowa City, IA).

\section{Antibodies}

The NF- $\kappa B$ pathway sampler kit (9936) was obtained from Cell Signaling (Danvers, MA), and antibodies were obtained from the following sources: anti-CD68 (MCA1957T) from Bio-Rad (Hercules, CA), anti-TLR2 (T2.5) from Invivogen (San Diego, CA), anti- $\alpha$-smooth muscle actin (A5228) from Sigma, anti-cleaved caspase-3 (AB3623MI) from Thermo Fisher Scientific (Waltham, MA), and antifibroblast specific protein 1 (FSP1; ab27957) and anti-Ki67 (ab15580) from Abcam (Cambridge, MA).

\section{Scoring of Tubule Injury}

Kidney sections were stained with periodic acid-Schiff by the pathology core at UT Southwestern Medical Center or at the University of Iowa; and for each sample, seven consecutive images were taken using a Zeiss (Oberkochen, Germany) Axioplan 2 deconvolution microscope. Tubule injury scores were assigned to periodic acid-Schiff-stained kidney sections ( $4 \mu \mathrm{m}$ thick) by an experienced kidney pathologist (P.R.) who was blinded to the identity of the samples. Published criteria were adopted, as described, for each parameter. ${ }^{22}$ Indicators of tubule injury/degeneration were vacuolization, intraluminal casts, and acellular/atrophic tubules. Scoring was as follows: 0 indicates none detected; $1,1 \%$ to $10 \%$ of tubules affected; $2,11 \%$ to $25 \%$ of tubules affected; $3,26 \%$ to $50 \%$ of tubules affected; and $4,>50 \%$ of tubules affected. Tubulointerstitial inflammation was defined by the presence of lymphocytes in perivascular and interstitial cortical areas. Scoring was as follows: 0 indicates no significant inflammation; 1 , foci in $1 \%$ to $10 \%$ of perivascular areas; $2,11 \%$ to $25 \%$ of the cortex affected; $3,26 \%$ to $50 \%$ of the cortex affected; and $4,>50 \%$ of the cortex affected.

\footnotetext{
Figure 2 Genetic deletion of Tlr2 reduces tubular damage, inflammation, and fibrosis in Glis2 knockout kidneys. A: Representative bright-field microscopy images of periodic acid-Schiff (PAS)-stained kidney sections at low (top row) and high (bottom row) magnification for Glis2, Tlr2 double-knockout $\left(\right.$ Glis2 $2^{-1-}$; Tlr2 $\left.{ }^{-/-}\right)$versus Glis2 knockout $\left(G l i s 2^{-1-}\right)$ mice. B-D: Comparison of kidney damage in kidney sections from the two genotypes, as assessed by PAS staining-based injury score (B), percentage of cystic area (calculated on the basis of digital image; C), and serum urea concentrations (D). E and F: Analysis of kidney fibrosis in both genotypes on the basis of Masson's trichrome staining. E: Kidney sections at low (top row) and high (bottom row) magnification. F: Quantification of trichrome staining by digital image analysis. G: Ratio of collagen/total protein content for kidneys of both genotypes. H-K: Analysis of inflammation in both genotypes. $\mathbf{H}$ and I: Representative immunofluorescence confocal images of kidney sections immunostained for the fibroblast marker fibroblast specific protein 1 (FSP1) (H) and the macrophage marker CD68 (I). J and K: Quantification of staining in $\mathbf{H}$ and $\mathbf{I}$, respectively, based on digital image analysis. $\mathbf{L}-\mathbf{N}$ : Comparison of markers of inflammation in the kidney, at the RNA level (quantitative real-time PCR). $P$ values were calculated by two-tailed $t$-test. Numbers of mice per group are reported in each panel. Data are expressed as means \pm SD $(\mathbf{B}-\mathbf{D}, \mathbf{F}, \mathbf{G}$, and $\mathbf{J}-\mathbf{N})$. ${ }^{*} P<0.05$, ${ }^{*} P<0.01$, and ${ }^{* * *} P<0.001$. Scale bars: $1 \mathrm{~mm}$ (A and $\mathbf{E}$, top row); $200 \mu \mathrm{m}$ (A and $\mathbf{E}$, bottom row); $20 \mu \mathrm{m}$ (H and $\left.\mathbf{I}\right)$. TNF- $\alpha$, tumor necrosis factor- $\alpha$.
} 
A
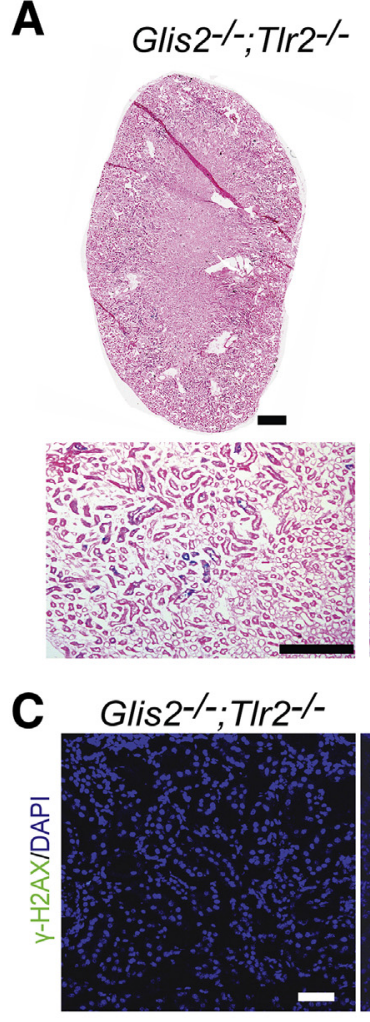

E
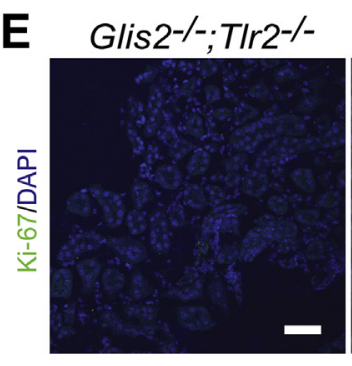

G

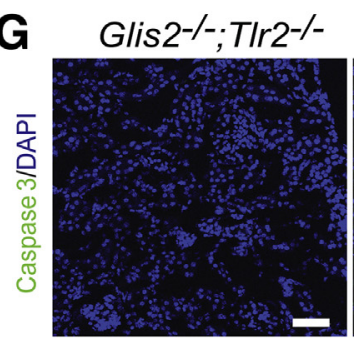

Glis2-/-
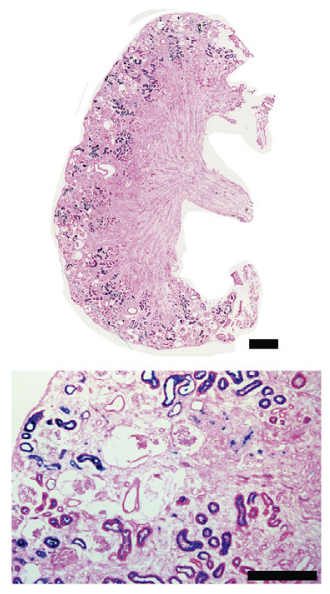

Glis2-/-

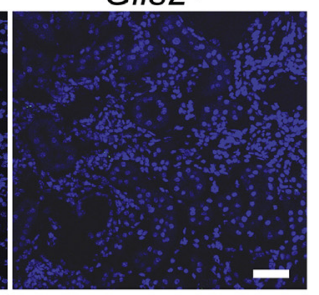

Glis2-/-
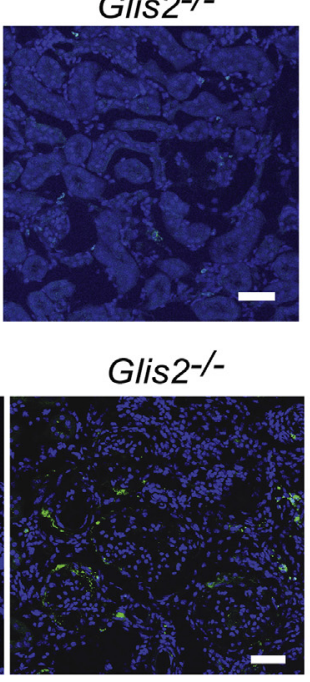

B
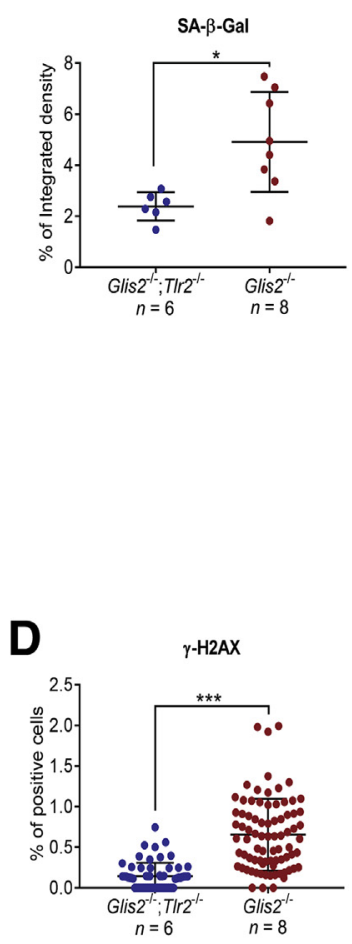

$\mathbf{F}$

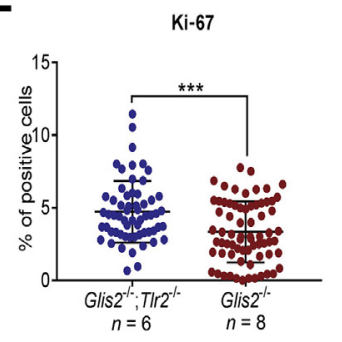

H

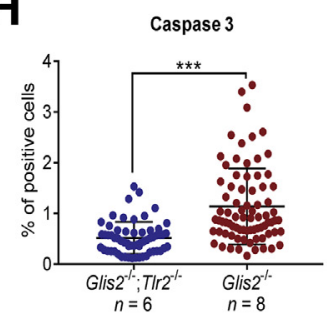

Figure 3 Genetic deletion of $T / r 2$ reduces levels of senescence in Glis2 knockout kidneys. A: Representative bright-field microscopy images of kidney sections showing senescence-associated $\beta$ galactosidase (SA- $\beta$-Gal) activity in kidneys of the Glis2, Tlr2 double-knockout and Glis2 knockout mice at low (top row) and high (bottom row) magnification. B: Comparison of signal in $\mathbf{A}$ between the two genotypes by digital image analysis. $\mathbf{C}-\mathbf{H}$ : Representative immunofluorescence confocal images of kidney sections of the two genotypes after immunostaining for the DNA damage marker $\gamma-\mathrm{H} 2 \mathrm{AX}(\mathbf{C})$, with the corresponding quantification (D); the proliferation marker Ki$67(\mathbf{E})$, with the corresponding quantification $(\mathbf{F})$; and the apoptosis marker activated caspase $3(\mathbf{G})$, with the corresponding quantification $(\mathbf{H}) . P$ values were calculated using the two-tailed $t$-test. Numbers of mice per group are reported in the panels. Data are expressed as means \pm SD (B, D, F, and $\mathbf{H}) .{ }^{*} P<0.05,{ }^{* * *} P<0.001$. Scale bars: 1 $\mathrm{mm}$ (A, top row); $200 \mu \mathrm{m}$ (A, bottom row); $20 \mu \mathrm{m}$ (C, E, and $\mathbf{G})$.

\section{Measurement of Serum Urea}

Serum was obtained as the supernatant from blood samples centrifuged at $1500 \times g$ for 10 minutes at room temperature. The serum urea concentration was determined using the Urea Assay Kit from Abcam (ab83362).

\section{Measurement of Ratio of Collagen/Total Protein}

Ten paraffin-embedded kidney sections $(10 \mu \mathrm{m}$ thick $)$ per mouse were hydrolyzed in $150 \mathrm{~mL} 6 \mathrm{~mol} / \mathrm{L} \mathrm{HCl}$, incubated for 20 hours at $95^{\circ} \mathrm{C}$, cooled, and centrifuged for 10 minutes at $16,000 \times g$. Concentrations of collagen and total protein in the supernatant were determined using collagen and protein assay kits from QuickZyme Biosciences (Leiden, the Netherlands).

\section{Immunofluorescence}

Kidneys were collected after mice were perfused with phosphate-buffered saline containing $4 \%$ paraformaldehyde, then fixed in $4 \%$ paraformaldehyde at $4{ }^{\circ} \mathrm{C}$ overnight, left in 
$18 \%$ sucrose solution in phosphate-buffered saline at $4^{\circ} \mathrm{C}$ overnight, and embedded in OCT compound. Frozen tissue sections $(10 \mu \mathrm{m}$ thick) were air dried for 30 minutes at room temperature and sequentially incubated in $0.1 \%$ Triton $\mathrm{X}-100$ solution in phosphate-buffered saline (20 minutes), $0.1 \% \mathrm{NaBH}_{4}$ solution (30 minutes), and blocking solution (1 hour) at room temperature. Tissues were incubated with primary antibody diluted in blocking solution overnight at $4^{\circ} \mathrm{C}$ and then with fluorescently labeled secondary antibody for 1 hour at room temperature. Samples were mounted using ProLong Gold Antifade Mountant with DAPI (Thermo Fisher Scientific, Waltham, MA). Images were acquired using either a Zeiss Axioplan 2 deconvolution microscope or a Zeiss LSM 710 confocal microscope with constant acquisition parameters.

\section{Measurement of Activity of SA- $\beta$-Gal}

Kidneys were collected and prepared as described above for immunofluorescence staining. Frozen sections ( $8 \mu \mathrm{m}$ thick) were air dried for 20 minutes and then incubated at $37^{\circ} \mathrm{C}$ for 12 to 16 hours in fresh senescence-associated $\beta$ galactosidase (SA- $\beta$-Gal) staining solution, which contains $1 \mathrm{mg} / \mathrm{mL}$ $\mathrm{X}$-Gal (stock solution in dimethylformamide; Teknova, Hollister, CA), $40 \mathrm{mmol} / \mathrm{L}$ citric acid/sodium phosphate (pH 6.0), $5 \mathrm{mmol} / \mathrm{L}$ potassium ferrocyanide, $5 \mathrm{mmol} / \mathrm{L}$ potassium ferricyanide, $150 \mathrm{mmol} / \mathrm{L} \mathrm{NaCl}$, and $2 \mathrm{mmol} / \mathrm{L}$ $\mathrm{MgCl}_{2}$. Sections were counterstained with eosin.

\section{Analysis of Digital Images}

Images of Western blot analyses, kidney sections stained using a protocol for immunofluorescence, SA- $\beta$-Gal, or Masson's trichrome were subjected to quantitative digital analysis. For each cortical region, 10 consecutive $\times 200$ optical fields per section were selected for measurement. Signal was quantified by digital image analysis using ImageJ software version 1.52c (NIH, Bethesda, MD; http:// imagej.nih.gov/ij).

\section{Quantitative Real-Time PCR}

Total RNA was extracted using the Qiagen RNeasy Mini kit. RNA was reverse transcribed using the iScript cDNA Synthesis Kit (Bio-Rad). Real-time PCR was performed using the CFX Connect Real-Time PCR Detection System (Bio-Rad) and iTaq Universal SYBR Green Supermix (Bio-Rad). All real-time PCR experiments were performed in triplicate. PCR primers were obtained from Integrated DNA Technology (Coralville, IA). Their sequences are the following: $\beta$-actin, $5^{\prime}$ GTACCACCATGTACCCAGGC-3' (forward) and $5^{\prime}$ AACGCAGCTCAGTAACAGTC- $3^{\prime}$ (reverse); IL-1 $\alpha, 5^{\prime}$ CGAAGACTACAGTTCTGCCATT-3' (forward) and $5^{\prime}$ GACGTTTCAGAGGTTCTCAGAG-3' (reverse); IL-1 $\beta, 5^{\prime}$ GCAACTGTTCCTGAACTCAACT-3' (forward) and $5^{\prime}$ ATCTTTTGGGGTCCGTCAACT-3' (reverse); and tumor necrosis factor-1 $\alpha, 5^{\prime}$-GCCTCTTCTCATTCCTGCTTG- $3^{\prime}$ (forward) and $5^{\prime}$-CTGATGAGAGGGAGGCCATT-3' (reverse).

\section{Statistical Analysis}

All data are presented as means \pm SD. Statistical significance $(P \leq 0.05$ was considered significant) was calculated using the two-tailed $t$-test or analysis of variance and Tukey's multiple comparisons test for more than two groups, using the SPSS17.0 software (IBM, Armonk, NY) or GraphPad Prism 7 (GraphPad Software, San Diego, CA).

\section{Results}

TLR2 Signaling Is Activated in Glis2 Knockdown Kidney Epithelial Cells and in Glis2 Knockout Kidneys

To gain insights into the molecular consequences of Glis2 loss in kidney epithelial cells, microarray-based differential expression analysis was performed, comparing immortalized mouse tubular cells stably expressing a Glis2-targeting shRNA. ${ }^{23}$ Counterpart cells stably expressing a shRNA targeting green fluorescent protein were used as a control. Many genes encoding components of the NF- $\kappa \mathrm{B}$ pathway, including $T l r 2$, were up-regulated in the Glis2-silenced but not control cells, suggesting that NF- $\kappa \mathrm{B}$ signaling is active in the absence of Glis2 (Figure 1A). Western blot analyses of cell lysates revealed that phosphorylation of serine 563 of the NF- $\kappa \mathrm{B}$ subunit $\mathrm{p} 65$, as well as of serine 32 of $\mathrm{I} \kappa \mathrm{B} \alpha$, was higher in the Glis2-silenced versus control cells, confirming

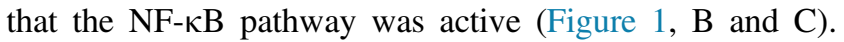
Supplementation of the culture medium with the TLR2 ligand lipopolysaccharide neither induced significant p65 phosphorylation in control cells nor further increased p65 phosphorylation in Glis2 knockdown cells. However, addition of a TLR2-blocking antibody (T2.5) resulted in a clear reduction of p65 phosphorylation in Glis 2 knockdown cells (Figure 1D). These findings indicate that Tlr2 contributes to NF- $\kappa \mathrm{B}$ activation in kidney tubular cells deficient for Glis2.

To assess the physiological relevance of these results, the consequences of loss of Glis2 were tested in vivo, comparing Tlr 2 activation in the kidney epithelial cells of the Glis2 knockout mouse with that in wild-type control animals. Examination of tubules within kidney sections by immunofluorescence confocal microscopy after staining with an antibody against Tlr2 revealed that, although in control samples the signal was localized at cell-cell junctions as expected, in the tubules of Glis2 knockout mice it was present in puncta throughout the cytoplasm (Figure 1, E and $\mathrm{F}$ ). This staining pattern is consistent with ligand-bound Tlr2 being activated and endocytosed in the Glis 2 knockout tubular cells in vivo. ${ }^{24}$

Collectively, these results indicate that the NF- $\kappa \mathrm{B}$ signaling pathway is activated in Glis2 knockout kidney 
A
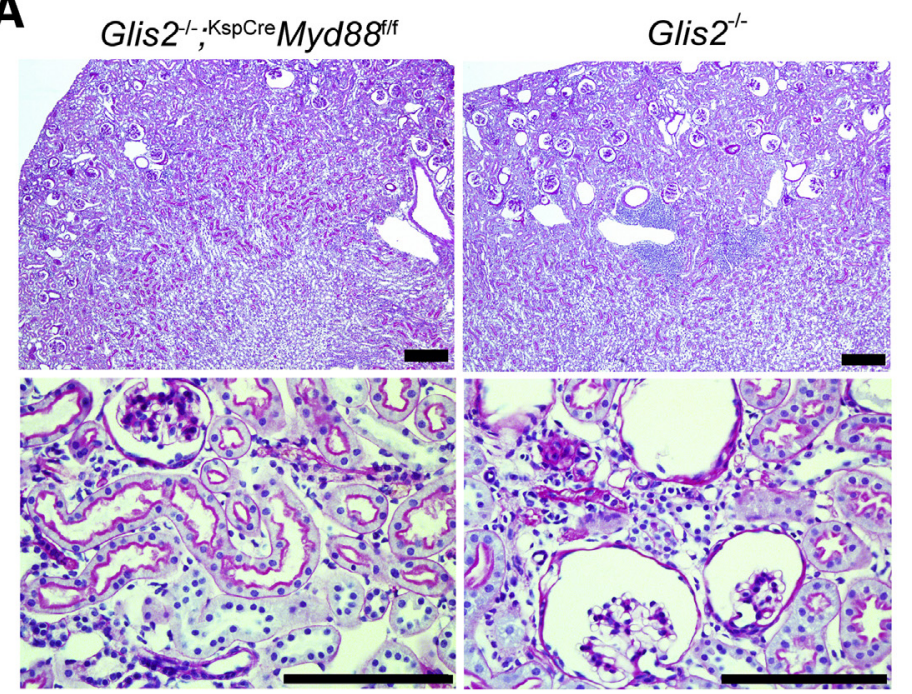

E
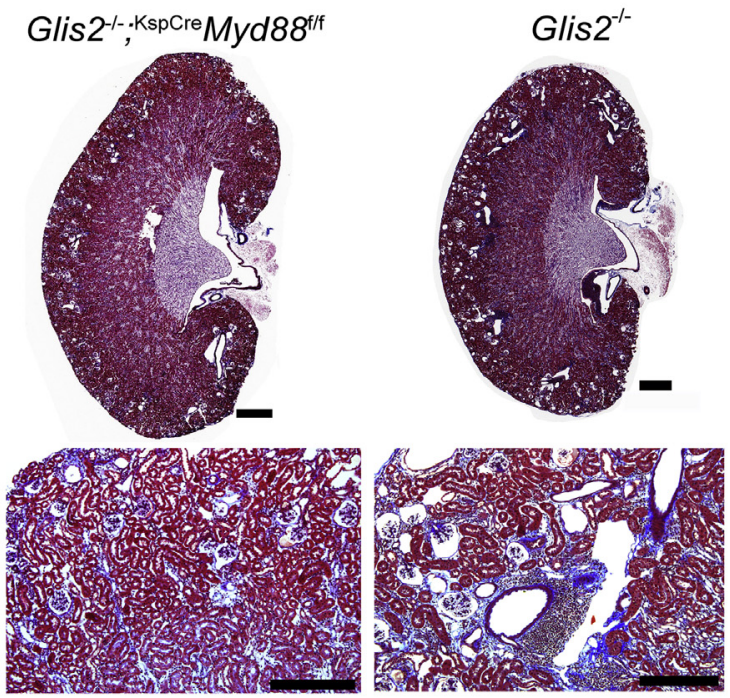

H
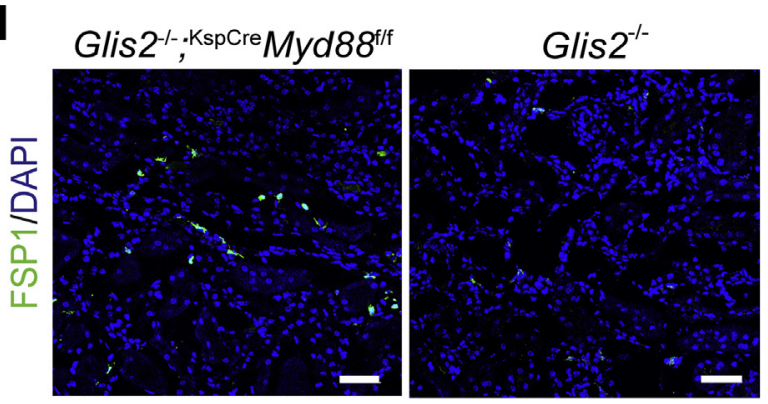

I
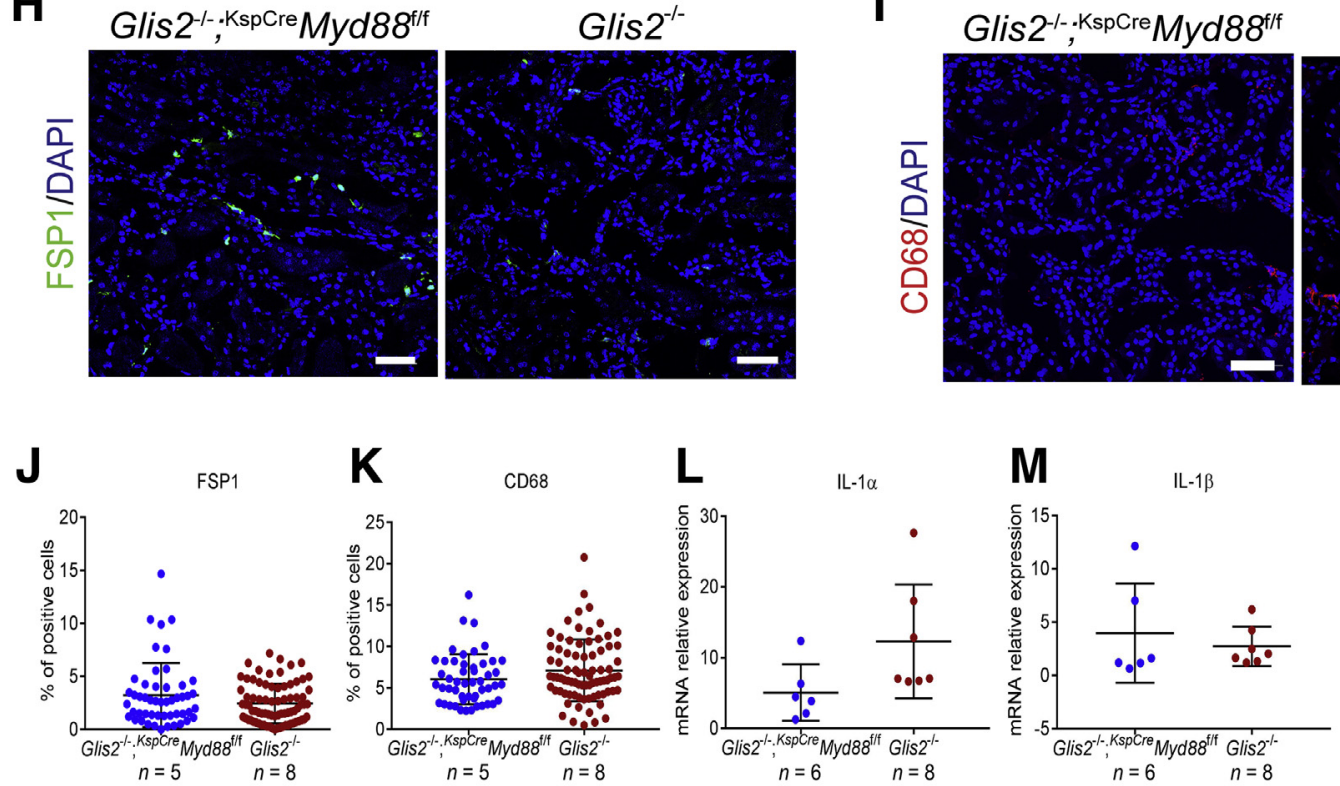

F

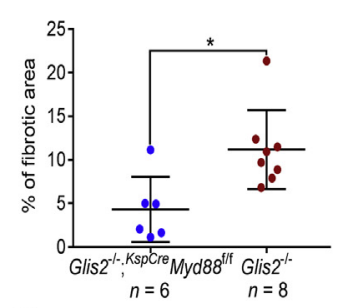

G

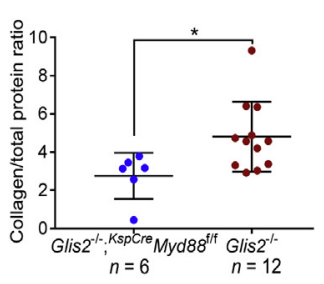

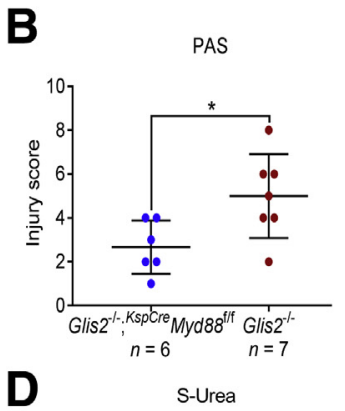

C

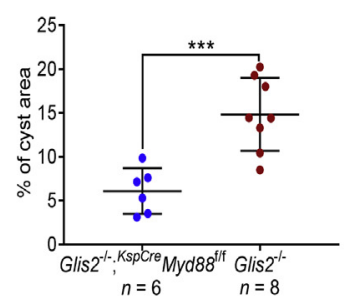


epithelial cells and that such activation is reinforced by signaling downstream of TLR2.

\section{Constitutive Genetic Deletion of Tlr2 Reduces Tubular Damage, Inflammation, and Fibrosis in Glis2 Knockout Kidneys}

On the basis of the results shown in Figure 1, we hypothesized that inactivation of signaling downstream of Tlr 2 might ameliorate the kidney phenotype of the Glis 2 knockout mice.

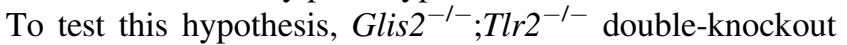
mice were generated and the resulting phenotype was compared with that of the Glis 2 knockout mice. This analysis was performed when the mice were 3 months of age, by which time point kidney damage and fibrosis have clearly manifested. $^{21,25}$ Analysis of bright-field microscopy images of kidney sections stained with periodic acid-Schiff (Figure 2A) revealed that tubule morphology (Figure 2B) and cystic dilation (Figure 2C) were less severe in the doubleknockout versus control mice. Renal function, assessed on the basis of serum urea concentrations, was also better in the double-knockout mice (Figure 2D). Examination of kidney sections by Masson's trichrome protocol (Figure 2E) showed that deletion of $T l r 2$ in mice deficient for Glis2 also protected against fibrosis (Figure 2, F and G), and digital quantification of immunofluorescence confocal microscopy images revealed that it prevented interstitial accumulation of fibroblasts (FSP-1-positive cells) and macrophages (CD68positive cells) (Figure 2, H-K). The expression of IL-1 $\alpha$ and tumor necrosis factor, but not IL- $1 \beta$ (Figure $2, \mathrm{~L}-\mathrm{N}$ ), was also lower in the double-knockout versus Glis2 knockout mice, indicating that a block of signaling downstream of Tlr2 prevents tubule damage, inflammation, and fibrosis that characterizes kidneys of the Glis 2 knockout mice.

\section{Genetic Deletion of Tlr2 Reduces Senescence and Apoptosis in Glis2 Knockout Kidneys}

Loss of Glis2 in kidney tubule cells causes activation of the replication stress response and extensive cell senescence. ${ }^{7}$ In senescent cells, activation of the NF- $\kappa \mathrm{B}$ pathway induces secretion of a wide array of proinflammatory molecules, including IL- $1 \alpha$, which signals through IL-1R on the recipient cells to promote senescence in the neighboring cells. ${ }^{8-10}$ Because the same signaling mechanisms result in activation of NF- $\kappa$ B downstream of both TLR2 and IL-1R, and these are further shared by TLR4, we hypothesized that the TLR/IL-1R signaling axis is responsible for a spread of the senescent phenotype in our mouse model of NPHP type 7. Our comparison of kidneys of the double-knockout versus Glis2 knockout mice showed that the activity of SA- $\beta-G a l$ in tubules was lower in the double-knockout versus Glis2 knockout kidneys (Figure 3, A and B). Consistent with this finding, the ratio of cells positive for the DNA damage marker $\gamma \mathrm{H} 2 \mathrm{AX}$ was lower, and that of the cells, tubular and interstitial, positive for the proliferation marker Ki-67 was higher in the double-knockout versus Glis2 knockout mice (Figure 3, $\mathrm{C}-\mathrm{F})$. The number of apoptotic cells, a feature of the Glis2 knockout kidney phenotype, ${ }^{25}$ was also lower in the doubleknockout kidneys (Figure 3, G and H).

\section{Epithelium-Specific Inactivation of Myd88 Protects Glis2 Knockout Kidneys from Tubular Damage and Fibrosis}

Tlr2, Tlr4, and IL-1R all signal through common downstream molecules, including Myd88. Tubule-specific deletion of Myd88 was sufficient to improve kidney damage, fibrosis, and tubular cell senescence in a mouse model of acute kidney injury. ${ }^{26}$ To determine whether the inactivation of Myd88 in epithelial cells would ameliorate the kidney phenotype of the Glis2 knockout, Glis2 knockout mice were generated in which both copies of the conditional allele Myd88 were knocked out in kidney tubular cells. The kidney-specific (KspCre) transgenic strain, in which Cre expression is driven by the promoter of the tubular cell-specific gene cadherin 16, was used. ${ }^{20}$ As in the case of our Glis2, Tlr2 double-knockout mice, the kidneys of Glis $2^{-1-}$; KspCre$M y d 88^{\text {ff/ }}$ mice had fewer signs of tubule damage: in images of sections of Glis $2^{-1-}$; KspCre $M y d 88^{\mathrm{f} / \mathrm{f}}$ kidneys stained with periodic acid-Schiff, fewer tubules were damaged, kidney atrophy was less pronounced, and the number of glomeruli with a dilated Bowman capsule was smaller (Figure 4, A-C). Serum concentrations of urea were also lower (Figure 4D). Concordant with this finding, collagen infiltration, as assessed by digital analysis of bright-field microscopy images of Masson's trichrome-stained kidneys, as well as the ratio of collagen/total protein were lower in Glis2 ${ }^{-1}$ ${ }^{-}$, KspCre $^{\mathrm{s}} \mathrm{Myd} 88^{\mathrm{f} / \mathrm{f}}$ than Glis2 single-knockout mice (Figure 4, $\mathrm{E}-\mathrm{G})$. However, the number of interstitial fibroblasts and

\footnotetext{
Figure 4 Epithelial cell-specific knockout of Myd88 reduces kidney damage and fibrosis but not inflammatory infiltration in Glis2 knockout kidneys. A: Representative bright-field microscopy images of periodic acid-Schiff (PAS)-stained kidney sections at low (top row) and high (bottom row) magnification for a mouse null for Glis2 and lacking Myd88 specifically in tubular cells (Glis $2^{-/-} ;$KspCre Myd88 $8^{\mathrm{fl} / \mathrm{fl}}$ ) versus the Glis2 knockout mouse. B-D: Comparison of kidney damage in kidney sections from the two genotypes, as assessed by PAS staining-based injury score (B), calculation of cystic area (C), and serum urea concentrations (D). E and F: Analysis of kidney fibrosis in both genotypes based on Masson's trichrome staining. Representative images of trichrome-stained kidney sections at low (top row) and high (bottom row) magnification (E). Quantification of trichome levels by digital image analysis (F). G: Ratios of collagen/total protein content for kidneys of both genotypes. H-K: Analysis of inflammation in both genotypes. Representative immunofluorescence confocal images of sections of kidneys immunostained for the fibroblast marker fibroblast specific protein 1 (FSP1) (H) and the macrophage marker CD68 (I) and quantification by digital image analysis $(\mathbf{J}$ and $\mathbf{K}$ ), respectively. $\mathbf{L}-\mathbf{N}$ : Comparison of markers of inflammation in the kidney, at the RNA level. $P$ values were calculated by two-tailed $t$-test. Numbers of mice per group are reported in the panels. Data are expressed as means \pm SD $(\mathbf{B}-\mathbf{D}, \mathbf{F}, \mathbf{G}$, and $\mathbf{J}-\mathbf{N})$. ${ }^{*} P<0.05$, ${ }^{* * *} P<0.001$. Scale bars: $1 \mathrm{~mm}$ (A and $\mathbf{E}$, top row); $200 \mu \mathrm{m}$ (A and $\mathbf{E}$, bottom row); $20 \mu \mathrm{m}$ (H and $\left.\mathbf{I}\right)$. TNF- $\alpha$, tumor necrosis factor- $\alpha$.
} 


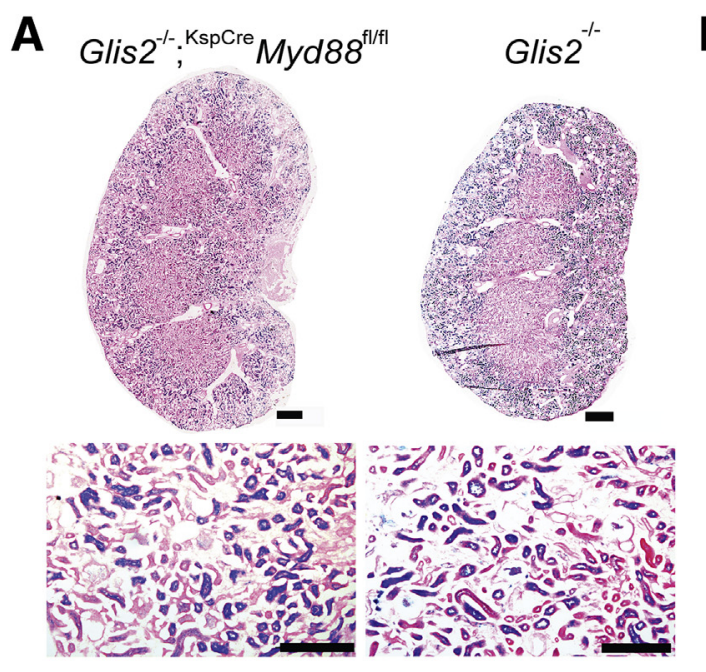

B
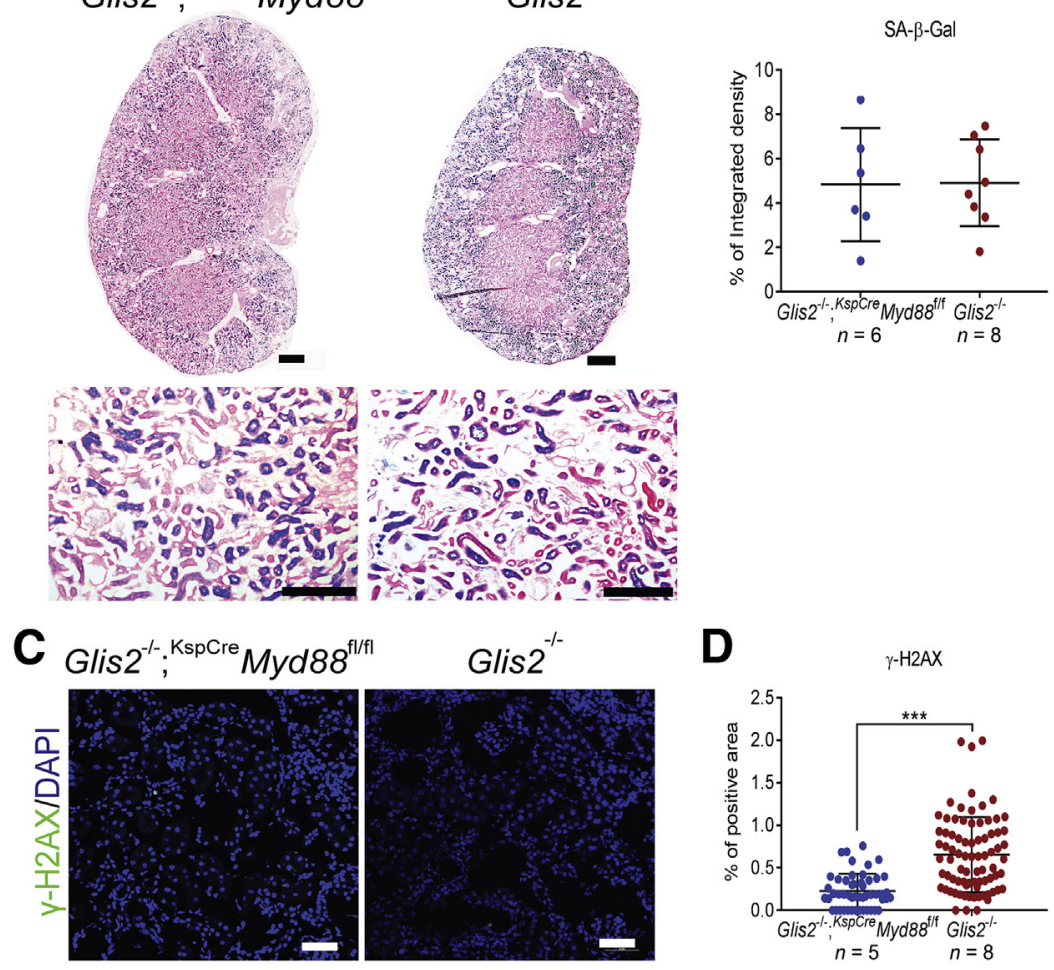

Glis2 $^{-1-}$

D
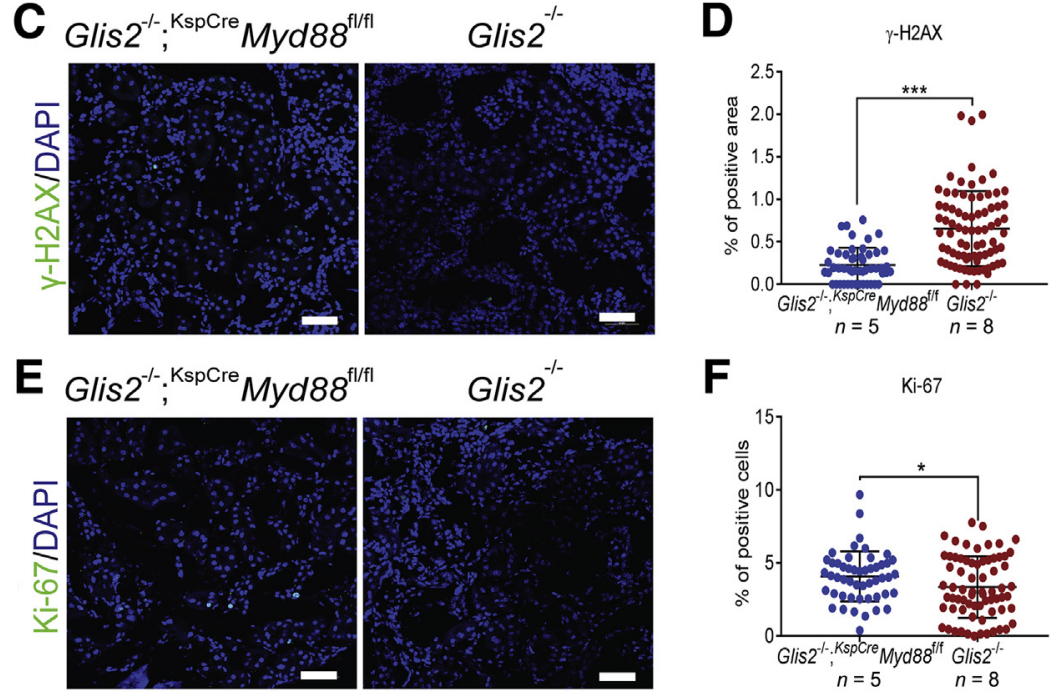

G Glis $^{-1-\text { KspCre }} \mathrm{Myd}^{\mathrm{f}} 8^{\mathrm{fl/f|}}$

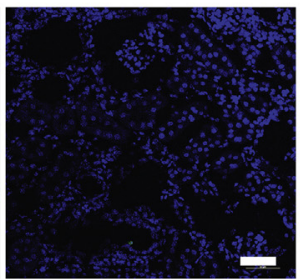

F

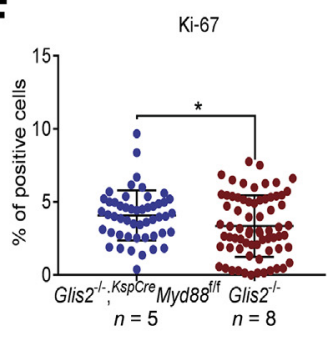

H

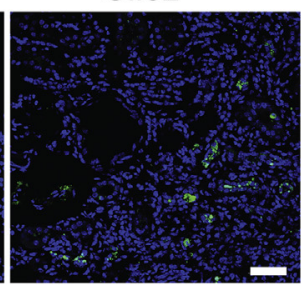

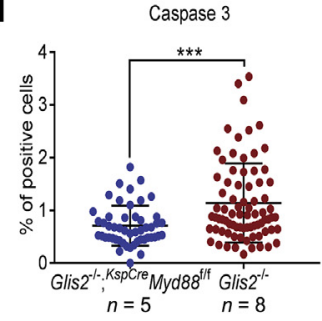

Figure 5 Epithelial cell-specific knockout of Myd88 in Glis2 knockout kidneys protects against DNA damage and apoptosis without affecting tubular cell senescence. A: Representative bright-field microscopy images of kidney sections showing senescence-associated $\beta$ galactosidase (SA- $\beta$-Gal) activity in a mouse null for Glis2 and lacking Myd88 specifically in tubular cells and in the Glis2 knockout mouse, at low (top row) and high (bottom row) magnification. B: Quantification of staining in A by digital image analysis. $\mathbf{C}-\mathbf{H}$ : Representative immunofluorescence confocal images for kidney sections from both genotypes, after immunostaining for: the DNA damage marker $\gamma-\mathrm{H} 2 \mathrm{AX}$ (C), with the corresponding quantification (D); the proliferation marker Ki-67 (E), with the corresponding quantification (F); and the apoptosis marker activated caspase $3(\mathbf{G})$, with the corresponding quantification $(\mathbf{H})$. $P$ values were calculated using the two-tailed $t$-test. Numbers of mice per group are reported in the panels. Data are expressed as means \pm SD (B, D, F, and $\mathbf{H})$. ${ }^{*} P<0.05,{ }^{* * *} P<0.001$. Scale bars: $1 \mathrm{~mm}$ (A, top row); $200 \mu \mathrm{m}$ (A, bottom row); $20 \mu \mathrm{m}$ (C, E, and $\mathbf{G}$ ). macrophages (Figure 4, H-K), as well as the levels of the inflammatory cytokines IL- $1 \alpha$, IL- $1 \beta$, and tumor necrosis factor- $\alpha$ (Figure $4, \mathrm{~L}-\mathrm{N}$ ), did not differ, suggesting that epithelium-specific deletion of Myd88 is only partially protective against the Glis2 knockout kidney phenotype.

Epithelium-Specific Inactivation of Myd88 in Glis2 Knockout Kidney Epithelial Cells Protects against DNA Damage and Apoptosis without Affecting Tubular Cell Senescence

To determine whether inactivation of Myd88 in tubular cells affects their senescence, the activity of SA- $\beta$-Gal was estimated and immunofluorescence microscopy was performed on kidney sections of Glis $2^{-/-} ;{ }^{\mathrm{KspCre}} M y d 88^{\mathrm{f} / \mathrm{f}}$ and Glis2 knockout mice using antibodies against Ki-67, a marker of actively cycling cells. Unlike the kidneys of the Glis2, Tlr2 double-knockout mice, those of the Glis2 ${ }^{-1}$ ${ }^{-} ;{ }^{\mathrm{KspCre}} M y d 88^{\mathrm{f} / \mathrm{f}}$ mice did not differ from those from Glis2 knockout kidneys in terms of SA- $\beta$-Gal activity, although the number of proliferating cells was slightly higher in the former (Figure 5, A and B). However, DNA damage (Figure 5, C and D) was lower in Glis $2^{-l-},{ }^{\mathrm{KspCre}} M y d 88^{\mathrm{f} / \mathrm{f}}$ versus Glis2 knockout kidneys. The number of proliferating cells (Figure 5, E and F) was slightly higher in the former, 
and the number of apoptotic cells (Figure 5, G and $\mathrm{H}$ ) was significantly lower in Glis $2^{-/-}$; ${ }^{\mathrm{KspCre}} M y d 88^{\mathrm{f} / \mathrm{f}}$ versus Glis2 knockout kidneys. These results indicate that epitheliumspecific inactivation of Myd88 in the absence of Glis2 provides partial protection from fibrosis and apoptosis, but at least in this model it does not significantly have an effect on tubular cell senescence.

\section{Pharmacologic Elimination of Senescent Cells Ameliorates Kidney Damage and Fibrosis, but Not Tubular Cell Senescence, in Glis2 Knockout Kidneys}

Although the precise details of the mechanisms that result in activation of the replication stress response in Glis2 knockout mice remain unclear, the presence of tubular cell senescence suggests that Nphp type 7 is a form of premature and progressive kidney aging. ${ }^{7}$ Because the elimination of senescent cells ameliorates organ inflammation in other models of chronic DNA damage and in the context of normal aging, ${ }^{27-31}$ it was determined whether the Glis2 knockout kidney phenotype can be ameliorated by pharmacologic elimination of senescent cells. Starting at 1 month of age, Glis2 knockout mice were injected intraperitoneally with the senolytic drug forkhead box protein O4 D-retro inverso (FOXO4-DRI), a synthetic peptide that induces apoptosis of senescent cells by disrupting the interaction between FOXO4 and $\mathrm{p} 53 .{ }^{29,32}$ Treatment was undertaken on alternate days during weeks $1,3,5$, and 7 after the beginning of the experiment; and the mice were sacrificed at 3 months of age, 7 days after the last dose of FOXO4-DRI (Figure 6A). At the end of the experiment, kidney pathology and renal function (Figure 6, B-E), interstitial fibrosis (Figure 6, F-H), and inflammation (Figure 6, I-O) were less severe in the treated versus control Glis 2 knockout mice, suggesting that senolytic drugs could represent an effective approach to reducing the inflammation and the fibrosis that characterizes NPHP. As expected, given the congenital nature of the molecular defect that drives senescence in Glis 2 knockout tubular cells, neither SA- $\beta$-Gal activity nor the proliferation index differed between FOXO4-DRI- and vehicle-treated control mice (Figure 7, A-D). However, both DNA damage and tubular cell apoptosis were reduced in the animals treated with FOXO4-DRI (Figure 7, E-H).

\section{Discussion}

NPHP encompasses a group of autosomal recessive chronic tubulointerstitial nephropathies that collectively are the most frequent genetic cause of chronic renal failure during the first three decades of life (up to $15 \%$ of cases of end-stage renal disease). No treatment is currently available, and NPHP patients inevitably progress to end-stage renal disease at a median age of 13 years. ${ }^{2,33}$

Mutations in many genes cause NPHP, and most of the encoded proteins localize to primary cilia, basal bodies, or centrosomes, cellular organelles that are strictly associated with the cell cycle. ${ }^{33,34}$ The centrosomes are the homes of several proteins that participate to DNA repair (DNA damage repair), and recent studies revealed an unexpected relationship between some proteins that cause ciliopathies (diseases caused by defects in proteins localized in the cilia) and cellular pathways that control DNA replication and repair. ${ }^{35,36}$ In addition, we recently showed that loss of function of Glis2 (Nphp7) is associated with DNA damage in kidney epithelial cells and with DNA damage repair activation that results in extensive cell senescence. ${ }^{7}$ Cell senescence is a protective mechanism evoked by multiple stressors, including DNA damage. Nevertheless, it is a double-edged sword. Despite its effectiveness in inhibiting neoplastic transformation ${ }^{37}$ and, in the short-term, its benefits with respect to tissue regeneration, ${ }^{32}$ it is detrimental in the long-term because it drives inflammation and age-related disease in multiple organs, including the kidney. ${ }^{27,38,39}$ This may be due to both the chronic proinflammatory effect of the SASP and a reduction in the regenerative capacity of tissues. ${ }^{40,41}$

NPHP shares many features with kidney aging, including thickening of the basal membrane, the formation of cysts, and atrophy of the tubules. ${ }^{42-44}$ The facts that the NF- $\mathrm{KB}$ signaling pathway is activated in Glis2-deficient kidney tubular cells both in vitro and in vivo and that this pathway mediates inflammation and other paracrine effects triggered by the SASP were our motivation for investigating whether the progression of kidney disease in Glis 2 knockout mice can be modulated by signaling downstream of TLRs/IL-1R and by targeting senescent cells with senolytic therapy. The findings from this study indicate that constitutive genetic deletion of $T l r 2$ and, to a lesser extent, tubule-specific deletion of Myd88 in Glis2 knockout mice not only were sufficient to improve tubular damage, kidney fibrosis, and inflammation, but also to reduce tubular cell's DNA damage and apoptosis in both mouse models. However, reduced cell senescence was only observed in Glis2, Tlr2 double knockouts but not in Glis $2^{-/-} ;{ }^{\mathrm{KspCre}} M y d 88^{\mathrm{f} / \mathrm{f}}$ mice.

Multiple factors may contribute to the differences in phenotype in these two mouse models. One might be the constitutive inactivation of $T l r 2$ in the double-knockout mice versus the tubule-specific deletion of Myd88 in their Glis $2^{-1-} ;{ }^{\mathrm{KspCre}} M y d 88^{\mathrm{f} / \mathrm{f}}$ counterparts. Given that $T l r 2$ is expressed largely in cells of the acquired and innate immune systems, it is possible that its constitutive inactivation has an additive effect, limiting proinflammatory signaling not only in tubular cells but also in immune interstitial cells, which are abundant in Glis2 knockout kidneys. ${ }^{21}$ In addition, it is possible that interstitial cells (pericytes, fibroblasts, and myofibroblasts) contribute prosenescence stimuli in a TLR-dependent manner; these stimuli would then be suppressed in the Glis2, Tlr2 doubleknockout mice. The observed differences may also be related to the level of expression of the KspCre transgene along the nephron: in fact, KspCre is more abundantly 
A

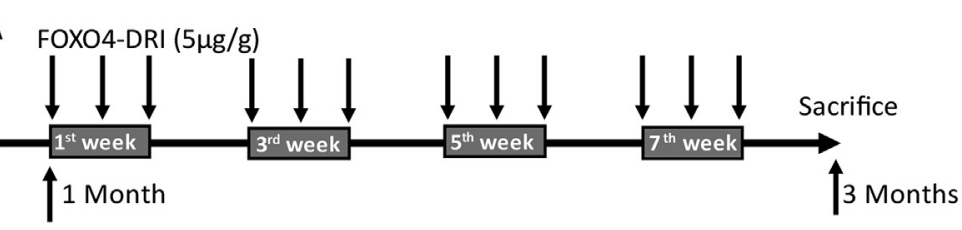

B

FOXO4-DRI

Vehicle

C

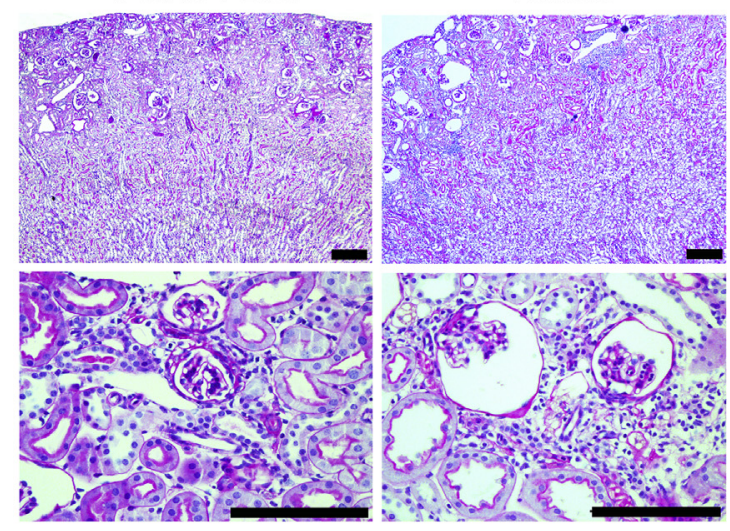

F
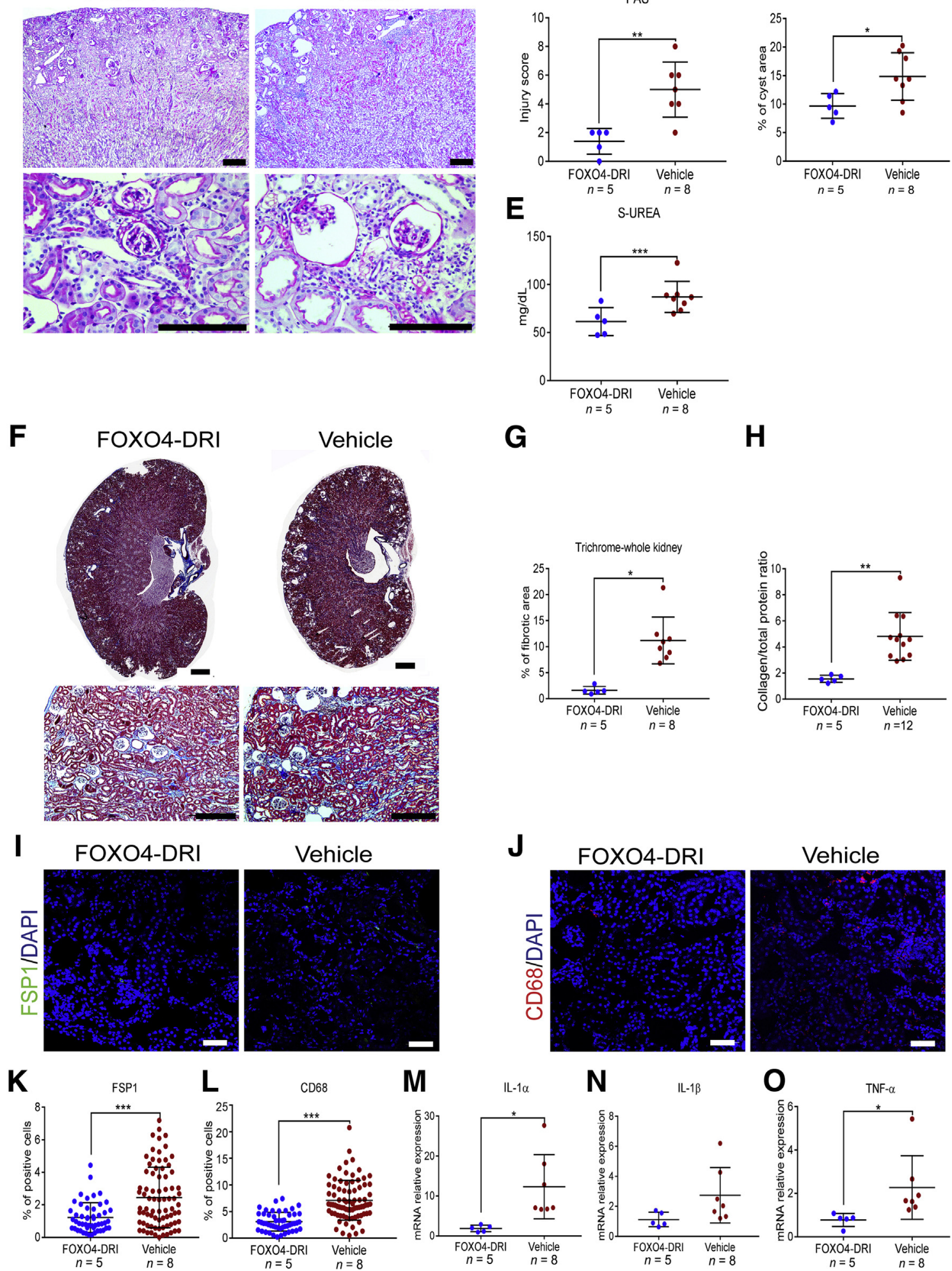

$E$

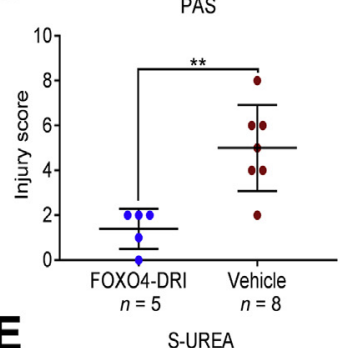

D

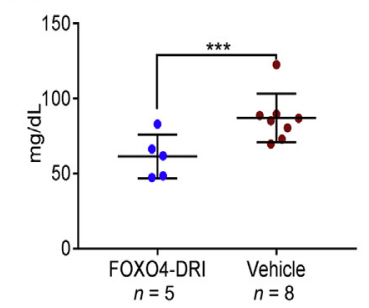

G

H
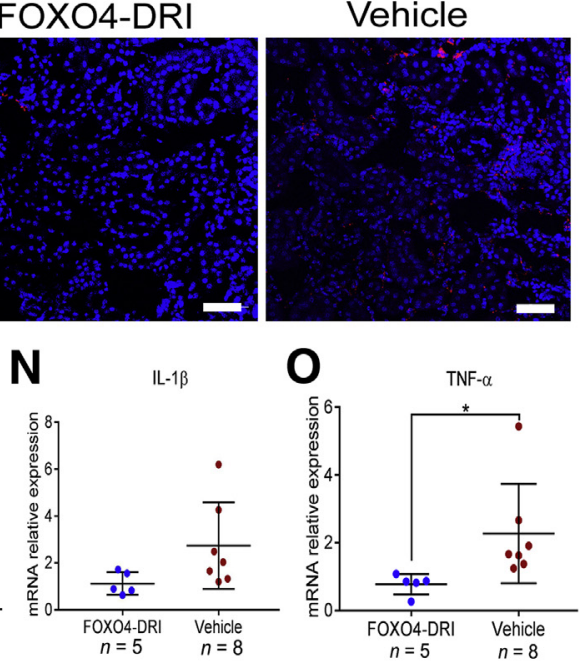


\section{A}

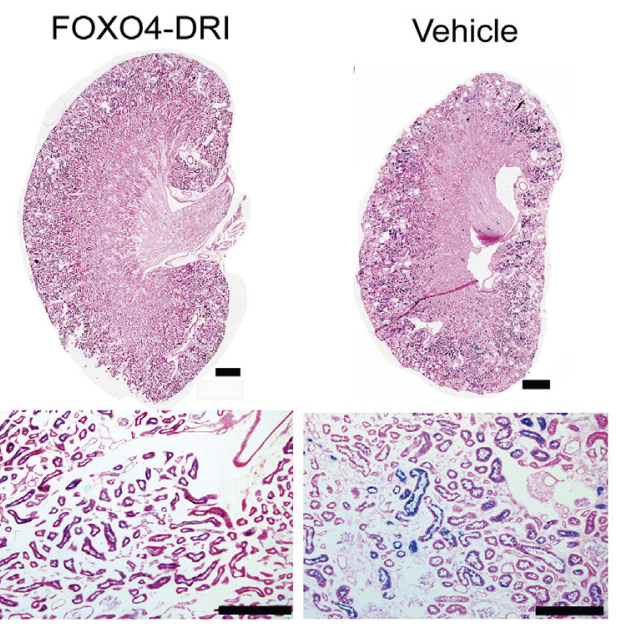

C

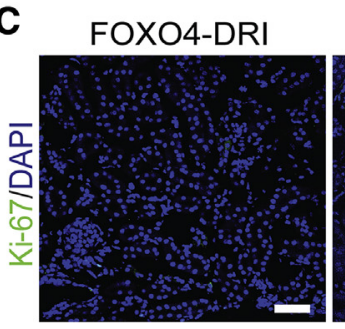

E

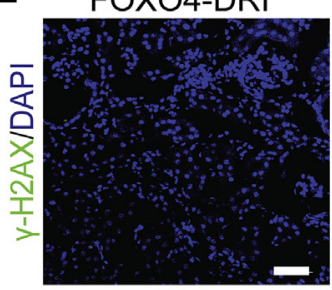

G

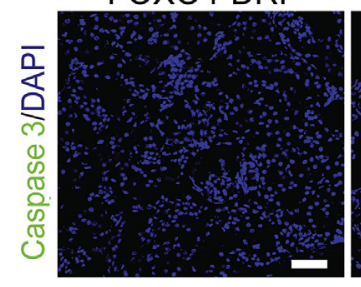

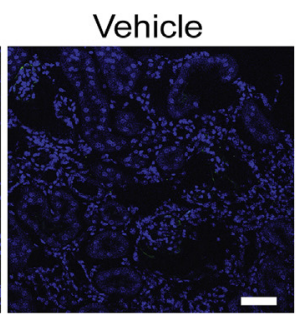

Vehicle

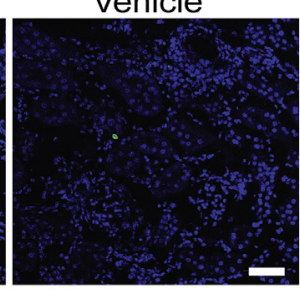

Vehicle

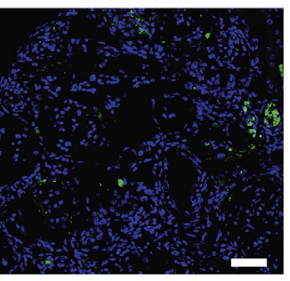

B

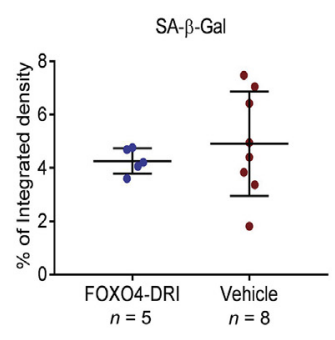

D

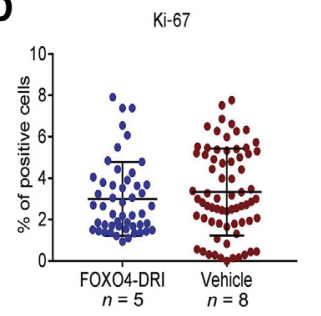

$\mathbf{F}$

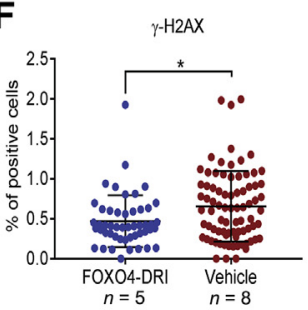

H

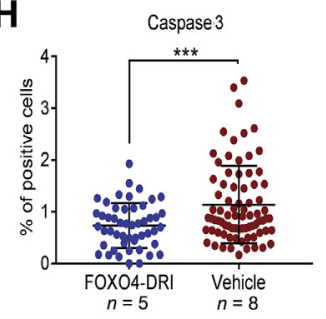

Figure 7 Treatment with the senolytic agent F0X04DRI does not significantly affect tubular cell senescence but decreases apoptosis in Glis2 knockout kidneys. A: Representative bright-field microscopy images of kidney sections showing senescence-associated $\beta$ galactosidase (SA- $\beta$-Gal) activity in Glis2 knockout mice treated with F0X04-DRI of vehicle, at low (top row) and high (bottom row) magnification. B: Quantification of staining in $\mathbf{A}$ by digital image analysis. $\mathbf{C}-\mathbf{H}$ : Representative immunofluorescence confocal images for kidney sections from both genotypes, after immunostaining for: the proliferation marker Ki-67 (C), with the corresponding quantification (D); the DNA damage marker $\gamma-\mathrm{H} 2 \mathrm{AX}(\mathbf{E})$, with the corresponding quantification (F); and the apoptosis marker activated caspase $3(\mathbf{G})$, with the corresponding quantification $(\mathbf{H}) . P$ values were calculated using the two-tailed $t$-test. Numbers of mice per group are reported in the panels. Data are expressed as means \pm SD (B, D, F, and H). ${ }^{*} P<0.05$, ${ }^{* * *} P<0.001$. Scale bars: $1 \mathrm{~mm}(\mathbf{A}$, top row); $200 \mu \mathrm{m}$ (A, bottom row); $20 \mu \mathrm{m}$ (C, E, and $\mathbf{G})$. expressed in the distal nephron but only sparsely in the proximal tubules, ${ }^{20}$ which is the compartment that is most affected in NPHP; thus, correction may be less effective in the Glis2 ${ }^{-/-} ;{ }^{\mathrm{KspCre}} M y d 88^{\mathrm{f} / \mathrm{f}}$ than in Glis2, Tlr2 doubleknockout mice. Future studies using other transgenic Cre lines are expected to clarify this point.

Figure 6 Treatment with the senolytic agent FOX04-DRI reduces kidney damage, inflammation, and fibrosis in Glis2 knockout kidneys. A: Schematic representation of the FOX04-DRI administration protocol used for the Glis2 knockout mice. Starting at 1 month of age, Glis2 knockout mice underwent i.p. injection with the senolytic drug FOX04-DRI on alternate days, during weeks 1, 3, 5, and 7 after initiation of the experiment, and they were sacrificed at 3 months of age. B: Representative bright-field microscopy images of periodic acid-Schiff (PAS)-stained kidney sections at low (top row) and high (bottom row) magnification for Glis2 knockout mice treated with FOX04-DRI or vehicle. C-E: Comparison of damage in kidney sections from the two treatment groups, as assessed by PAS staining-based injury score (C), percentage of cystic area, as calculated by digital image analysis (D), and serum urea concentrations (E). F and G: Analysis of fibrosis defects in both treatment groups based on Masson's trichrome staining. F: Representative images of trichrome-stained kidney sections at low (top row) and high (bottom row) magnification, for mice treated as in A. G: Quantification of trichome levels in $\mathbf{F}$ by digital image analysis. H: Ratio of collagen/total protein content in kidneys of mice treated as in F. Analysis of inflammation in both treatment groups. I and $\mathbf{J}$ : Representative immunofluorescence confocal images of sections of kidneys for the fibroblast marker fibroblast specific protein 1 (FSP1) (I) and the macrophage marker CD68 (J). K and L: Quantification of marker expression in $\mathbf{I}$ and $\mathbf{J}$, respectively, by digital image analysis. $\mathbf{M}-\mathbf{0}$ : Analysis of inflammation in both treatment groups, at the RNA expression level. $P$ values were calculated by two-tailed $t$-test. Numbers of mice per group are reported in the panels. Data are expressed as means $\pm \mathbf{S D}(\mathbf{C}-\mathbf{E}, \mathbf{G}, \mathbf{H}$, and $\mathbf{K}-\mathbf{0})$. ${ }^{*} P<0.05$, ${ }^{*} P<0.01$, and ${ }^{* * *} P<0.001$. Scale bars: $1 \mathrm{~mm}$ (B and $\mathbf{F}$, top row); $200 \mu \mathrm{m}$ (B and $\mathbf{F}$, bottom row); $20 \mu \mathrm{m}$ (I and $\mathbf{J}$ ). TNF- $\alpha$, tumor necrosis factor- $\alpha$. 
Apart from the differences between the two mouse crosses that were studied, proliferation was increased and DNA damage and apoptosis were significantly less severe after both constitutive inactivation of $T l r 2$ and tubulespecific inactivation of Myd88. The increased proliferation and the reduced apoptosis in Glis $2^{-1-} ;{ }^{\mathrm{KsCre}} M y d 88^{\mathrm{f} / \mathrm{f}}$ mice suggest that TLR/IL-1R signaling has a cell-autonomous effect on tubular cell survival and imply that its inhibition might be beneficial not only through the limitation of proinflammatory activity of the SASP, but also through ameliorating the regenerative capacity of the Glis2-defective tubular epithelial cells.

The extensive tubular cell senescence that affects kidneys of the Glis 2 knockout mice is consistent with the notion that NPHP is a form of premature and progressive kidney aging, ${ }^{7}$ similar to other genetic diseases of premature aging that have been shown to be secondary to chronic DNA damage. $^{29,39}$ As in the latter, it is possible that NPHP patients would benefit from senolytic therapy. ${ }^{27-31}$ Therefore, the effect of senolysis was tested in a mouse model of NPHP type 7. Indeed, the pharmacologic deletion of senescent cells via the administration of FOXO4-DRI indicated a clear positive effect of such a strategy with respect to tubular damage, inflammation, and fibrosis. These effects were most likely a consequence of a reduction in the accumulation of SASP components derived from senescent tubular and, perhaps, interstitial cells. Despite these positive effects, the levels of SA- $\beta$-Gal activity and the proliferation index of the tubular cells were unaffected by the senolytic therapy. A possible interpretation of these results, given the congenital nature of the defect that causes senescence in Glis2 knockout tubular cells, is that after the cessation of FOXO4DRI therapy, more Glis $2^{-/-}$tubular cells enter senescence. In this case, a therapeutic approach based on senolysis would likely be only palliative, yet it may prolong the lifespan of the kidney and delay the need for dialysis in patients affected by NPHP. Future studies will clarify if alternative therapeutic regimens or the use of different types of senolytic agents will be able to optimize the attenuation of kidney damage in the Glis2 mouse model of NPHP.

In conclusion, these results indicate that signaling from the TLR/IL-1R receptors of the innate immune system contributes to the progression of tubule damage, inflammation, and fibrosis in the Nphp 7 mouse model of nephronophthisis and that the suppression of such signaling improves tubular cell proliferation, DNA damage, and apoptosis. In addition, these findings suggest that the elimination of senescent cells might be useful in delaying the progression of kidney disease in this and other forms of NPHP.

\section{References}

1. Wolf MT: Nephronophthisis and related syndromes. Curr Opin Pediatr 2015, 27:201-211

2. Luo F, Tao YH: Nephronophthisis: a review of genotype-phenotype correlation. Nephrology (Carlton) 2018, 23:904-911
3. Chaki M, Airik R, Ghosh AK, Giles RH, Chen R, Slaats GG, et al: Exome capture reveals ZNF423 and CEP164 mutations, linking renal ciliopathies to DNA damage response signaling. Cell 2012, 150: $533-548$

4. Choi HJ, Lin JR, Vannier JB, Slaats GG, Kile AC, Paulsen RD, Manning DK, Beier DR, Giles RH, Boulton SJ, Cimprich KA: NEK8 links the ATR-regulated replication stress response and $S$ phase CDK activity to renal ciliopathies. Mol Cell 2013, 51: 423-439

5. Airik R, Slaats GG, Guo Z, Weiss AC, Khan N, Ghosh A, Hurd TW, Bekker-Jensen S, Schroder JM, Elledge SJ, Andersen JS, Kispert A, Castelli M, Boletta A, Giles RH, Hildebrandt F: Renal-retinal ciliopathy gene Sdccag8 regulates DNA damage response signaling. J Am Soc Nephrol 2014, 25:2573-2583

6. Slaats GG, Saldivar JC, Bacal J, Zeman MK, Kile AC, Hynes AM, Srivastava S, Nazmutdinova J, den Ouden K, Zagers MS, Foletto V, Verhaar MC, Miles C, Sayer JA, Cimprich KA, Giles RH: DNA replication stress underlies renal phenotypes in CEP290-associated Joubert syndrome. J Clin Invest 2015, 125:3657-3666

7. Lu D, Rauhauser A, Li B, Ren C, McEnery K, Zhu J, Chaki M, Vadnagara K, Elhadi S, Jetten AM, Igarashi P, Attanasio M: Loss of Glis2/NPHP7 causes kidney epithelial cell senescence and suppresses cyst growth in the Kif3a mouse model of cystic kidney disease. Kidney Int 2016, 89:1307-1323

8. Acosta JC, Banito A, Wuestefeld T, Georgilis A, Janich P, Morton JP, Athineos D, Kang TW, Lasitschka F, Andrulis M, Pascual G, Morris KJ, Khan S, Jin H, Dharmalingam G, Snijders AP, Carroll T, Capper D, Pritchard C, Inman GJ, Longerich T, Sansom OJ, Benitah SA, Zender L, Gil J: A complex secretory program orchestrated by the inflammasome controls paracrine senescence. Nat Cell Biol 2013, 15:978-990

9. Tchkonia T, Zhu Y, van Deursen J, Campisi J, Kirkland JL: Cellular senescence and the senescent secretory phenotype: therapeutic opportunities. J Clin Invest 2013, 123:966-972

10. Chien Y, Scuoppo C, Wang X, Fang X, Balgley B, Bolden JE, Premsrirut P, Luo W, Chicas A, Lee CS, Kogan SC, Lowe SW: Control of the senescence-associated secretory phenotype by NFkappaB promotes senescence and enhances chemosensitivity. Genes Dev 2011, 25:2125-2136

11. Campisi J: Cellular senescence: putting the paradoxes in perspective Curr Opin Genet Dev 2011, 21:107-112

12. Herranz N, Gil J: Mechanisms and functions of cellular senescence J Clin Invest 2018, 128:1238-1246

13. Orjalo AV, Bhaumik D, Gengler BK, Scott GK, Campisi J: Cell surface-bound IL-1alpha is an upstream regulator of the senescenceassociated IL-6/IL-8 cytokine network. Proc Natl Acad Sci U S A 2009, 106:17031-17036

14. Kawai T, Akira S: Signaling to NF-kappaB by Toll-like receptors Trends Mol Med 2007, 13:460-469

15. Ve T, Gay NJ, Mansell A, Kobe B, Kellie S: Adaptors in toll-like receptor signaling and their potential as therapeutic targets. Curr Drug Targets 2012, 13:1360-1374

16. Johnson CM, Tapping RI: Microbial products stimulate human Tolllike receptor 2 expression through histone modification surrounding a proximal NF-kappaB-binding site. J Biol Chem 2007, 282: 31197-31205

17. Coppe JP, Desprez PY, Krtolica A, Campisi J: The senescenceassociated secretory phenotype: the dark side of tumor suppression. Annu Rev Pathol 2010, 5:99-118

18. Janssens S, Tschopp J: Signals from within: the DNA-damageinduced NF-kappaB response. Cell Death Differ 2006, 13:773-784

19. McCool KW, Miyamoto S: DNA damage-dependent NF-kappaB activation: NEMO turns nuclear signaling inside out. Immunol Rev 2012, 246:311-326

20. Shao X, Somlo S, Igarashi P: Epithelial-specific Cre/lox recombination in the developing kidney and genitourinary tract. J Am Soc Nephrol 2002, 13:1837-1846 
21. Kim YS, Kang HS, Herbert R, Beak JY, Collins JB, Grissom SF, Jetten AM: Kruppel-like zinc finger protein Glis2 is essential for the maintenance of normal renal functions. Mol Cell Biol 2008, 28: $2358-2367$

22. Hur E, Garip A, Camyar A, Ilgun S, Ozisik M, Tuna S, Olukman M, Narli Ozdemir Z, Yildirim Sozmen E, Sen S, Akcicek F, Duman S: The effects of vitamin d on gentamicin-induced acute kidney injury in experimental rat model. Int J Endocrinol 2013, 2013:313528

23. Li B, Rauhauser AA, Dai J, Sakthivel R, Igarashi P, Jetten AM, Attanasio M: Increased hedgehog signaling in postnatal kidney results in aberrant activation of nephron developmental programs. Hum Mol Genet 2011, 20:4155-4166

24. Brandt KJ, Fickentscher C, Kruithof EK, de Moerloose P: TLR2 ligands induce NF-kappaB activation from endosomal compartments of human monocytes. PLoS One 2013, 8:e80743

25. Attanasio M, Uhlenhaut NH, Sousa VH, O'Toole JF, Otto E, Anlag K, Klugmann C, Treier AC, Helou J, Sayer JA, Seelow D, Nurnberg G, Becker C, Chudley AE, Nurnberg P, Hildebrandt F, Treier M: Loss of GLIS2 causes nephronophthisis in humans and mice by increased apoptosis and fibrosis. Nat Genet 2007, 39: $1018-1024$

26. Jin $\mathrm{H}$, Zhang $\mathrm{Y}$, Ding $\mathrm{Q}$, Wang SS, Rastogi $\mathrm{P}$, Dai DF, Lu D, Purvis M, Cao C, Wang A, Liu D, Ren C, Elhadi S, Hu MC, Chai Y, Zepeda-Orozco D, Campisi J, Attanasio M: Epithelial innate immunity mediates tubular cell senescence after kidney injury. JCI Insight 2019, 4. e125490

27. Baker DJ, Childs BG, Durik M, Wijers ME, Sieben CJ, Zhong J, Saltness RA, Jeganathan KB, Verzosa GC, Pezeshki A, Khazaie K, Miller JD, van Deursen JM: Naturally occurring p16(Ink4a)-positive cells shorten healthy lifespan. Nature 2016, 530:184-189

28. Farr JN, Xu M, Weivoda MM, Monroe DG, Fraser DG, Onken JL, Negley BA, Sfeir JG, Ogrodnik MB, Hachfeld CM, LeBrasseur NK, Drake MT, Pignolo RJ, Pirtskhalava T, Tchkonia T, Oursler MJ, Kirkland JL, Khosla S: Targeting cellular senescence prevents agerelated bone loss in mice. Nat Med 2017, 23:1072-1079

29. Baar MP, Brandt RMC, Putavet DA, Klein JDD, Derks KWJ, Bourgeois BRM, Stryeck S, Rijksen Y, van Willigenburg $H$, Feijtel DA, van der Pluijm I, Essers J, van Cappellen WA, van IWF, Houtsmuller AB, Pothof J, de Bruin RWF, Madl T, Hoeijmakers JHJ, Campisi J, de Keizer PLJ: Targeted apoptosis of senescent cells restores tissue homeostasis in response to chemotoxicity and aging. Cell 2017, 169:132-147.e16

30. Serrano M: Senescence helps regeneration. Dev Cell 2014, 31 : 671-672
31. Ritschka B, Storer M, Mas A, Heinzmann F, Ortells MC, Morton JP, Sansom OJ, Zender L, Keyes WM: The senescence-associated secretory phenotype induces cellular plasticity and tissue regeneration. Genes Dev 2017, 31:172-183

32. Demaria M, Ohtani N, Youssef SA, Rodier F, Toussaint W, Mitchell JR, Laberge RM, Vijg J, Van Steeg H, Dolle ME, Hoeijmakers JH, de Bruin A, Hara E, Campisi J: An essential role for senescent cells in optimal wound healing through secretion of PDGFAA. Dev Cell 2014, 31:722-733

33. Hildebrandt F, Attanasio M, Otto E: Nephronophthisis: disease mechanisms of a ciliopathy. J Am Soc Nephrol 2009, 20:23-35

34. Badano JL, Teslovich TM, Katsanis N: The centrosome in human genetic disease. Nat Rev Genet 2005, 6:194-205

35. Zhang S, Hemmerich P, Grosse F: Centrosomal localization of DNA damage checkpoint proteins. J Cell Biochem 2007, 101:451-465

36. Attanasio M: Ciliopathies and DNA damage: an emerging nexus. Curr Opin Nephrol Hypertens 2015, 24:366-370

37. Serrano M, Lin AW, McCurrach ME, Beach D, Lowe SW: Oncogenic ras provokes premature cell senescence associated with accumulation of p53 and p16INK4a. Cell 1997, 88:593-602

38. Baker DJ, Perez-Terzic C, Jin F, Pitel KS, Niederlander NJ, Jeganathan K, Yamada S, Reyes S, Rowe L, Hiddinga HJ, Eberhardt NL, Terzic A, van Deursen JM: Opposing roles for p16Ink4a and p19Arf in senescence and ageing caused by BubR1 insufficiency. Nat Cell Biol 2008, 10:825-836

39. Baker DJ, Wijshake T, Tchkonia T, LeBrasseur NK, Childs BG, van de Sluis B, Kirkland JL, van Deursen JM: Clearance of p16Ink4apositive senescent cells delays ageing-associated disorders. Nature 2011, 479:232-236

40. Garcia-Prat L, Martinez-Vicente M, Perdiguero E, Ortet L, Rodriguez-Ubreva J, Rebollo E, Ruiz-Bonilla V, Gutarra S, Ballestar E, Serrano AL, Sandri M, Munoz-Canoves P: Autophagy maintains stemness by preventing senescence. Nature 2016, 529:37-42

41. Bernet JD, Doles JD, Hall JK, Kelly Tanaka K, Carter TA, Olwin BB: p38 MAPK signaling underlies a cell-autonomous loss of stem cell selfrenewal in skeletal muscle of aged mice. Nat Med 2014, 20:265-271

42. Denic A, Glassock RJ, Rule AD: Structural and functional changes with the aging kidney. Adv Chronic Kidney Dis 2016, 23:19-28

43. Rule AD, Sasiwimonphan K, Lieske JC, Keddis MT, Torres VE, Vrtiska TJ: Characteristics of renal cystic and solid lesions based on contrast-enhanced computed tomography of potential kidney donors. Am J Kidney Dis 2012, 59:611-618

44. Darmady EM, Offer J, Woodhouse MA: The parameters of the ageing kidney. J Pathol 1973, 109:195-207 